\title{
Nearly Inviscid Faraday Waves
}

\author{
Edgar Knobloch \\ José M. Vega
}

\begin{abstract}
Many powerful techniques from Hamiltonian mechanics are available for the study of ideal hydrodynamics. This articie explores some of the consequences of including small viscosity in a study of surface gravitycapillary waves excited by the vertical vibration of a container. It is shown that in this system, as in others, the addition of snatl viscosity provides a singular perturbation of the ideal fluid problem, and that as a result its effects are nontrivial. The relevance of existing studies of ideal fluid problerns is discussed from this point of view.
\end{abstract}

\section{Contents}

1 Introduction ................. I81

2 The Faraday System. . . . . . . . . . . . 185

3 Gravity-Capillary Woves in Moderately Large

Aspect-13.atic Contenjurs . . . . . . . . . . . 194

4 Dynamics of the Reduced Frjuations. . . . . . . . 200

4.1 Two-Mode Modol and Basic Solutions . . . . . . 201

4.2 Numerical Results ... . . . . . . . . . 204

4.3 Comparison with the PDE ........... 211

5 Concluding Remarks ............... 215

References ..................... 219

\section{Introduction}

Jerry Marsden has been a driving force in studies of ideal hydrodynamics using methods from Hamilionjan mechanics. Perhaps his most important contribution has been the discovery of a systematic procedure for the construction of noncanonical Hamiltonian structures for such flows. The required noncanonical Poisson brackets are typically singular, implying the 
presence of additional conserved quantities known as Casimirs. Using these techniques he and his colleagues were able to extend Arnol'd-type stability theorems to a number of flows of importance in geophysics and engineering (Marsden and Morrison [1984]; Holm, Marsden, Ratiu, and Weinstein [1985]; Abarbanel, Holm, Marsden, and Ratiu "1986]; Holm, Marsden, and Ratiu [1986]; Lewis, Marsden, Montgomery, and Ratiu [1986]). These are major contributions to the field of ideal hydrodynamics and are nowadays taught in graduate level courses on the subject.

Although these techniques are powerful, and enable one to obtain results that would be hard to obtain by other meaus, there remains an important question as to their relevance to flows in the real world. Unless one studies flows in a superfluid, for example ${ }^{4} \mathrm{He}$ below the $\lambda$-point (i.e., at temperatures below the transition to superfluidity) such flows are inevitably affected by dissipative processes, be they viscous or thermal. These may have an importance beyond being responsible for the decay of the flow on the slow diffusive time scale (Batchelor [1967]: Chorin and Marsden [1979]). In general the presence of small viscosity is responsible for the formation of thin boundary layers where the flow departs drastically from that in the bulk. In such boundary layers worticity is generated by viscous effects and this vorticity may then diffuse or be convected into the bulk. In such cases the flow in the bulk may be substantially modified. Boundary layers may be classified as passive or dynamic, depending on their effect on the bulk flow. Passive boundary layers do not affect the flow in the bulk, which will then resemble the potential solution over long times; such boundary layers serve merely to adjust the flow to the physically relevant boundary conditions. In the absence of homdary liwe sepratation sucts boundary lizyers are found, for example, in steady flow aroukd pbstarios. Oscillatory boundary laycrs may likewise be passive if the oscillation aistplitude is smiall and only the? leading order oxcillatory flow is consislered. However as discussed further below, this is no longer so at second order in amplitude. In this and other cases the boundary layess can becurne dynamic, and force the flow in the bulk even though this flow remains largely inviscid. In such cases the inviscid flow in the bulk differs substantially from the flow that would be obtained by ignoring the boundary layers altogether, and this effect persists in the limit in which the viscosity ranishes, i.e., in these cases the limit of vanishing viscosity may have at most a tenuous connection with the behavior of the strictly inviscid system (Batchelor [1967]). The present article is devoted to the explication of this phenomenon in the context of a particularly interesting plysical system, gravity-capillary waves in a vertically vibrating container (the Faraday system).

The difference between the properties of the Euler equation for an ideal incompressible fluid and the Navier-Stokes equation in the limit of large Reynolds number provides the most famous example of the dangers of ignoring viscosity entirely, in the sense that the 'thermodynamic equilibrium' spectrum that results bears no relation to the energy spectrum in 
the so-called inertial range. But there are simple examples of problems not involving turbulence where viscosity, however small, also plays a profound role. Perhaps the simplest is provided by the computation of the Lagrangian drift of a fluid element when a surface gravity-capillary wave passes overhead. This drift is important because its sum over all the fluid elements may be identified with the linear momentum associated with the wave (Knobloch and Pierce [1998], and references therein). In the following we employ Cartesian coordinates: with the $x$-axis along the unpcrturbed free surface of the fluid and $y$ vertically upwards. An irrotational incompressible flow then satisfies the equation

$$
\nabla^{2} \phi=0
$$

where $\mathbf{u} \equiv\left(\phi_{x}, \phi_{y}\right)$ is the Eulerian vclocity, subject to the boundary conditions

$$
\begin{array}{cl}
\phi_{y}=0 \quad \text { at } y=-h \\
f_{t}+\phi_{x} f_{x}=\phi_{y}: \quad \phi_{t}+|\mathbf{u}|^{2} / 2+p / \rho+g f=0 \quad \text { at } y=f .
\end{array}
$$

Here $f$ is the free surface deflection: $p=p_{0}-T f_{x x}\left(1+f_{x}^{2}\right)^{-3 / 2}$, the excess pressure being a consequence of the presence of the surfice tension $T$, and $g$ and $\rho$ are, respectively, the acceleration due to gravity and the fluid density. A formulation of this type assumes that the fluid remains irrotational if it is irrotational initially. This is so only if the fluid is strict?y inviscid.

Since a particle starting at $\mathbf{x}=\mathbf{a}$ at $t=0$ is at

$$
\mathbf{x}=\mathbf{a}+\int_{0}^{t} \mathbf{v}\left(\mathbf{a}, t^{\prime}\right) d t^{\prime}
$$

at time $t$, the Lagrangian velocity of the flud element at time $t$ is given, to second order, by

$$
\mathbf{v}(\mathbf{a}, t)=\mathbf{u}(\mathbf{a}, t)+\left(\int_{0}^{t} \mathbf{u}\left(\mathbf{a}, t^{\prime}\right) d t^{\prime}\right) \cdot \nabla_{\mathbf{a}} \mathbf{u}(\mathbf{a}, t)
$$

For a progressive sinusoidal wave of (small) amplitude $A, f=A \cos (k x-$ $\omega t)+O\left(A^{2}\right)$ and $\phi=\left[f_{t} \cosh k(y+h)\right] / k \sinh k h+O\left(A^{2}\right)$. If $A$ is constant in space it is possible to show that the time-averaged Eulerian velocity $\langle\mathbf{u}\rangle$ vanishes to second order but the time-averaged Lagrangian drift $\langle\mathbf{v}\rangle$ does not:

$$
\langle v\rangle=\left(\frac{\mu k A^{2} \cosh 2 k(y+h)}{2 \sinh ^{2} k h_{2}}, 0\right) .
$$

This drift is known as the Stokes drift. However, in the presence of small viscosity, this result is misleading. The argument that follows goes back to the work of Schlichting [1932. Observe tłat for sufficiently small viscosity (namely $\beta h \gg 1, \beta / k \gg 1$, where $\beta=(\omega / 2 \nu)^{1 / 2}$ ) the inviscid solution 
applies everywhere except in the two thin oscillatory viscous boundary layers of $O\left(\beta^{-1}\right)$ thickness along the top artd bottom, whose contribution can be superposed on top of the irrotational flow just computed. Therefore, if in the bottom boundary layer we write $\mathbf{u}=\nabla \phi+\mathbf{u}^{\prime}$, then at leading order $\mathbf{u}^{\prime}=\left(u^{\prime}, v^{\prime}\right)$ satisfies the linearized vorticity equation

$$
\frac{\partial \Omega}{\partial t}=\nu\left(\frac{\partial^{2} \Omega}{\partial x^{2}}+\frac{\partial^{2} \Omega}{\partial y^{2}}\right): \quad \Omega=\nabla \times \mathbf{u}^{\prime},
$$

subject to the boundary conditions

$$
u^{\prime}=-\phi_{x}: \quad y^{s}=0 \text { at } y=-h ; \quad u^{\prime}=0 \text { for } \beta(y+h) \gg 1 .
$$

This problem has the solution

$$
\begin{aligned}
& u^{\prime}=-\omega A \operatorname{cosech} k h \mathrm{e}^{-\beta[y+h)} \cos (k x-\omega t+\beta(y+h)), \\
& v^{\prime}=-\int_{-h}^{y}\left[\partial u^{\prime}(x, z, t) / \partial x\right] d z .
\end{aligned}
$$

With these expressions it is possible to compute a time-averaged Reynolds stress in the oscillatory boundary layer:

$$
\left\langle u^{\prime} v^{\prime}\right\rangle=\frac{\omega^{2} k A^{2}}{4 \beta \sinh ^{2} k h}\left[2(\beta \tilde{y} \sin \beta \tilde{y}+\cos \beta \tilde{y}) \mathrm{e}^{-\beta \tilde{y}}-\mathrm{e}^{-2 \beta \tilde{y}}-1\right],
$$

correct to second order in the wave amplitude $A$. Here $\tilde{y} \equiv y+h$. This Reynolds stress drives a mean flow $\left(C^{\prime}(y), 0\right\}$ according the mean moment.um equation $v \partial^{2} U^{\prime} / \partial y^{2}-\partial\left(u^{\prime} v^{\prime}\right) / \partial y$, i.e.,

$$
\nu^{\partial u^{\prime}}=\left\langle u^{\prime} v^{\prime}\right\rangle\left\langle u^{\prime}\right\rangle_{\infty}
$$

where $\left\langle u^{\prime} v^{\prime}\right\rangle_{\infty}$ represents the Reynolds stress just outside of the boundary layer. Letting $\beta(y+h) \rightarrow \infty$ one finds that.

$$
\left\langle u^{\prime} u_{\infty}=-\omega^{2} k A^{2} /\left(4 \beta \sinh ^{2} k f_{h}\right)\right. \text {. }
$$

In view of the requirement $V^{r}(-h)=0$ equation (1.5) now implies that

$$
U^{\prime}(y) \rightarrow U_{\infty}^{\prime}=\frac{3 \omega k A^{2}}{4 \sinh ^{2} k h} \quad \text { for } \beta(u+h) \gg 1 .
$$

Thus the time-averaged Eulerian velocity at the edge of the boundary layer is (a) finite at second order, and (b) independent of $\nu$ (for sufficiently small $\nu$ ), provided only that $\nu>0$ ! Since this Eulerian mean flow also carrics the fluid elements with it its effect must be added to the Stokes drift (1.2) computed on the basis of inviscid theory. Thus the net Lagrangian drift for $\beta h \gg 1, \beta / k \gg 1$ is in fact

$$
\langle\mathbf{v}\rangle_{\infty}=\left(\frac{5 \omega k A^{2}}{4 \sinh ^{2} k h}, 0\right)
$$


a value that is $5 / 2$ times the inviscid value (Longuet-Higgins [1953]; Batchelor [1967]; Phillips [1977]; Craik [1982]]. As recognized already by LonguetHiggins [195:3], a somewhat similar effect is present at the free surface as well. It is clear therefore that the oscillatory viscous boundary layers must be retained even in the limit of arbitrarily small viscosity, and that these are effective at driving large scale mean flows even when the viscosity $\nu$ is arbitrarily small.

In the following wre discuss in some detail the corresponding phenomena in the Faraday system, where oscillatory viscous boundary layers are incvitably present, and explore the interaction between the Faraday instability and the mean flow driven in these boundary layers. In systems of small to moderate aspect ratio such mean flows are entirely of viscous origin (Nicolás and Vega [1996; Higuera; Nicolás, and Vega [2000]), but in the larger aspect ratio systems of intercst. below the situation is rather more subtle because of the presence of an additional inniscid mean fiow. For inviscid free waves this mean flow is associated with spatial modulation of a single mode, as described by the cclebrated Davey-Stewartson equations (Davey and Stewartson [1974]: Pierce and Knobloch [1994]). If viscosity is retained and the system forced, as in a shear flow, a similar set of equations but with conıplex cocfficients can be derived (Davey, Hocking, and Stewartson [1974]). In general the mean flow present will contain both viscous and inviscid contributions, even in nearly inviscid flows. It is because of these effects that one cannot mimic the effects of viscosity on an oscillating fluid systcm by simply adding dissipation post facto to an otherwise inviscid theory.

\section{The Faraday System}

Surface gravity-capillary wawes excited parametrically by the vertical oscillation of a container provide a convenient and well-studied system (Miles and Henderson [1990]; Fauve [1995]; Kudrolli and Gollub [1997]), where the issues raised in the preceding section come to the fore. We nondimensionalize distances with the unperturbed depth $h$ and time with the gravity-capillary time $\left[g / h-T /\left(\rho h^{3}\right)^{-1 / 2}\right.$. In two dimensions the resulting viscous problem is then described by the dimensionless equations (Vega, Knobloch, and Martel [201]1),

$$
\begin{gathered}
\psi_{x x x}+\psi_{y y}=\Omega, \quad \Omega_{t}-\psi_{y} \Omega_{x}+\psi_{x} \Omega_{y}=C_{g}\left(\Omega_{x x}+\Omega_{y y}\right), \\
f_{t}-\psi_{x}-\psi_{y} f_{x}=\left(\psi_{y y}-\psi_{x x}\right)\left(\mathbf{1}-f_{x}^{2}\right)-4 f_{x} \psi_{x y}=0 \text { at } y=f, \\
(1-S) f_{x}-S\left(f_{x} / \sqrt{1+f_{x}^{2}}\right)_{x x}-\psi_{y x}+\psi_{x t} f_{x}-\left(\psi_{x}+\psi_{y} f_{x}\right) \Omega \\
\quad+\left(\psi_{x}^{2}+\psi_{y}^{2}\right)_{x} / 2+\left(\psi_{x}^{2}+\psi_{y}^{2}\right)_{y} f_{x} / 2-4 \psi_{i}^{2} \cos (2 \omega t) f_{x} \\
=-C_{y}\left[3 \psi_{x x y}+\psi_{y y y}-\left(\psi_{x x x}+\psi_{x y y}\right) f_{x}\right]
\end{gathered}
$$




$$
\begin{aligned}
&+2 C_{g}\left[\frac{2 \psi_{x y} f_{x}^{2}-\left(\dot{\psi}_{x x}-\dot{\psi}_{y y}\right) f_{x}}{1+f_{x}^{2}}\right]_{x} \\
&+2 C_{g} \frac{\left(\psi_{x x y}-\psi_{y y y}\right) f_{x}^{2}-\psi_{x y y}\left(1-f_{x}^{2}\right\} f_{x}}{1+f_{x}^{2}} \text { a. } y=f, \\
& \int_{0}^{L} \Omega_{y} d x=\psi=\psi_{y}=0 \text { at } y=-1: \quad \int_{0}^{L} f d x=0,
\end{aligned}
$$

where $\psi$ is the streamfunction, defined such that $\mathbf{u} \equiv\left(-\psi_{y}, \psi_{x}\right)$ is the velocity, $\Omega \equiv \nabla \times \mathbf{u}$ is the vorticity, and $f$ is again the free surface deflection. The latter is required to satisfy rolume conservation as in $(2.4 \mathrm{~d})$. In an annular container of dimensionless length $L$ periodic boundary conditions are applied to all quantities: in this case the boundary condition (2.4a) guarantees that the pressure is also periodic in $x$. The resulting problem depends on

$$
\begin{array}{ll}
L & \text { the aspect ratio, } \\
\mu & \text { the nondimensional vibration amplitude, } \\
2 \omega & \text { the nondimensional vibration frequency, } \\
C_{g}=\nu /\left[g h^{3}+(T h / \rho)\right]^{1 / 2} & \text { the capillary-gravity namber, } \\
S=T /\left(T+\rho g h^{2}\right) & \text { the gravity-capillary balance parameter. }
\end{array}
$$

Here $\nu$ is the kinematic viscosity. Thus $C_{g}$ and $S$ are related to the usual capillary number $C=\nu[\rho / T h]^{1 / 2}$ and the Bond number $B=\rho g h^{2} / T$ by

$$
C_{g}=C /(1+B)^{1 / 2} \text { and } S=1 /(1+B)
$$

Note that $0 \leq S \leq 1$ and that the extreme walues $\$-0,1$ correspond to the purely gravitabional $(T=0)$ and the purcly capillary $(y=0)$ limits, respectivcly.

The formulation employed abowe nses the streanfunction wy and not the velocity potential $\phi$, since formulations of the Faraday problem in terms of the latter miss both the mechanism for tite gemeration of (Fulerian) mean flows already discussed in $\S 1$, and the possibility that vorticity will diffuse from the viscous boundary layers along walls and the frec surface into the nominally inviscid interior. These boundary layers form because in the presence of viscosity the tangential velocity must vanish along any wall while the tangential stress along the free surface is also required to vanish. Neither of these wo effects is restored by the a posteriori addition of damping to a fundamentally inviscid formulation, i.e., a formulation based on the velocity potential. In fact. for times that are not too long the vorticity contamination of the bulk does remain regligible, so that the flow in the bulk is correctly described by an inviscid formulation but with boundary conditions determined by a boundary layer analysis as in $\$ 1$.

The basic assumption made below is that viscosity is small, namcly

$$
C_{g} \ll \mathbf{1}
$$


However, as already mentioned, this does not mean that viscous effects can be safely ignored. Indeed. the subtleties arise already at the level of the linear problem. The normal modes of the unforced problem, linearized around $\psi=f=0$, take the form

$$
\left(\psi_{i}: f\right)=(\Psi, F) \mathrm{e}^{\lambda t+i_{k} x} .
$$

In the limit (2.5) there are two bypes of such modes (Kakutani and Matsuuchi [1975]; Martel and Knobloch [1997]):

A. The nearly inviscid modes (or surface modes) obcy the dispersion relation

$$
\lambda=\mathrm{i} \omega-(1-\mathrm{i}) \alpha_{1} C_{g}^{1 / 2}-\alpha_{2} C_{g}+O\left(C_{g}^{3 / 2}\right),
$$

where

$$
\begin{aligned}
\omega & =\left[k \sigma\left(1-S-S k^{2}\right)\right]^{1 / 2}, \\
\alpha_{1} & =\frac{k(\omega / 2)^{1 / 2}}{\sinh (2 k)} \\
\alpha_{2} & =\frac{k^{2}}{4 \sigma^{2}}\left(1+8 \sigma^{2}-\sigma^{4}\right)
\end{aligned}
$$

and $\sigma \equiv \tanh k$. Eq. (2.T) prowides a good approximation for both the frequency $\pm \operatorname{Im}(\lambda)$ and the damping rate;

$$
\hat{d} \equiv-\operatorname{Re}(\lambda)=\alpha_{1} C_{g}^{1 / 2}+\alpha_{2} C_{3}
$$

for small but fixed values of $C_{\Omega}$, see Fig. 2.1. Hawever, as noted in Martel and Knobloch [1997), if the (corrected) third term in (2.7) is ornitted the resulting approximation breaks down as soon as $k \geq k_{m} \sim\left|\ln C_{g}\right|$. Since these moderately larwe values of $k$ are also of interest this term is retaincd irl what follows.

The eigenfunction associated with the disporsion rclation (2.7) is given (up to a constant, factors) by

$$
(\Psi, F)=\left(\Psi_{0}, 1\right)+O\left(C_{g}^{1 / 2}\right) . \quad \Psi_{0}=\frac{\omega \sinh [k(y+1)]}{k \sinh k} .
$$

These modes therefore exhibit a significant free-surface deflection; moreover, they are irrotational in the bulk: outside two thin boundary layers of thickness $O\left(\left(C_{g} / \omega\right)^{1 / 2}\right)$ attached to the bottom plate and the free surface. Since the decay rate of these modes is $O\left(C_{g}^{1 / 2}\right)$ for small $C_{g}$ these modes are near-marginal in nearly inviscid fluids. Note that the horizontal wavenumber $k$ is only restricted by the periodicity condition and thus can take any value of the form $2 \pi N / L$, where $N$ is an integer; in the limit $L \rightarrow \infty$ the allowed wavenumbers become dense on the real line. In the following we assume that the basic disturbance consists of a pair of counterpropagating wavetrains with wavenumber $I k$ and frequency $w$ determined from the above dispersion relation: and that the mean flow arises from nonlinear 

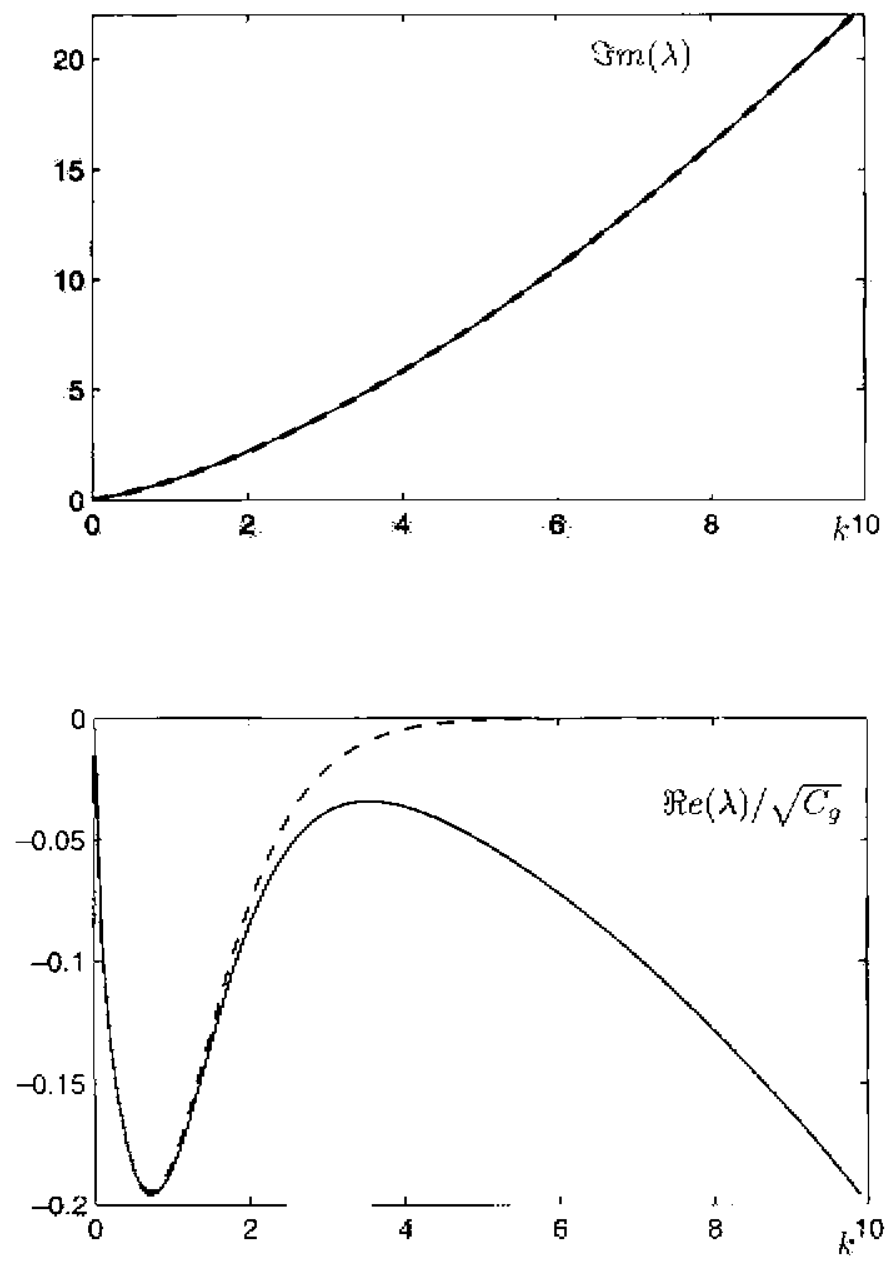

FiguRE 2.1. The nearly inviscid dispersion relation, $\Im m \lambda$ and $\Re e \lambda$ vs. $k$, for $C_{g}=10^{-6}, S=0.5$, from Eq-(2.T) using fine $O\left(C_{g}^{1 / 2}\right)$ results (dashed line) and the $O\left(C_{g}\right)$ results (soljd line). These paranieters correspond to the experiments of Henderson and Miles [1994].

interactions involving these two modes. Here $i$ represents half the forcing frequency. Thus the relewant nearly intiscid modes are either of long wavelength $(k \rightarrow 0)$ or are concentrated around the two counterpropagating modes. The long wave modes constitute the nearly inviscid mean flow; in the strictly inviscid case, this flow is the mean flow considered in inviscid theories (Davey and Stewartson [1974]; Pierce and Knobloch [1994]). However, because of its long wavelength this mean flow does not appear if the aspect ratio is of order unity (Nicolás and Vega [1996]; Higuera, Nicolás, 
and Vega $[2000])$.

$B$. The viscous modes (or hydrodynamical modes) obey the dispersion relation

$$
\lambda=-C_{g}\left(k^{2}+q_{n}(k)^{2}\right]+O\left(C_{g}^{2}\right)
$$

where for each $k>0, q_{n}>0$ is the $n$-th root of $q \tanh k=k$ tan $q$, and hence decay on an $O\left(C_{g}\right)$ timeseale, i.e., more slowly than the surface modes when $C_{g}$ is sufficiently small. Consequently these modes are also near-marginal. Since the associated eigenfunction is

$$
\Psi=\sin q_{n} \sinh (k y)-\sinh k \sin \left(q_{n} y\right)-O\left(C_{g}\right), \quad F=O\left(C_{g}\right),
$$

these modes do not result in any significant free-surface deformation at leading order. On the other hand they are rotational throughout the domain and, when forced at the edge of the oscillatory boundary layers along the bottom (Schlichting [1932]) and the free surface (Longuct-Higgins [1953]) by the mechanism described in $\$ 1$, they constitute the viscous mean flow. In vicw of its slow decay this flow must be included in any realistic nearly inviscid description.

With this in mind it is now possible to perform a multiscale analysis of the viscous fluid equations using $C_{g}, L^{-1}$ and $\mu$ as unrelated small parameters. We focus on two well-separated scales in both space $(x \sim 1$ and $x \gg$ 1) and time $(t \sim 1$ and $t \gg 1)$, and derive equations for small, slowlyvarying amplitudes $A$ and $B$ of left- and right-propagating waves. Since viscosity is small, we must distinguish three regions in the physical domain, namely, the two oscillatory boundary layers (of thickress $O\left(C_{g}^{1 / 2}\right)$ ) and the remaining part (or butk) of the domain (see Fig. 2.2). The boundary layers Inust be consilemed in orrier to obtain the correct boundary conditions for the solution in the bulk. The details of the derivation are quite involved and can be fourd in a rocent paper (Woga, Knobloch, and Martel [2001]), where explicit conditions for the validity of the resulting equations as a description of the two-dimensional uearly inviscid Faraday system are also derived. The resulting equations take the form

$$
\begin{aligned}
A_{t}-v_{g} A_{x}= & \mathrm{i} \alpha A_{x x}-(\delta+\mathrm{i} d) A+\mathrm{i}\left(\left.\alpha_{3}\left|A^{2}-\alpha_{4}\right| B\right|^{2}\right) A+\mathrm{i} \alpha_{5} \mu \bar{B} \\
& +\mathrm{i} \alpha_{65} \int_{-1}^{0} g(y)\left(\psi_{y}^{m}\right)^{x} \mathrm{~d} y A+\mathrm{i} \alpha_{7}\left(f^{m}\right)^{x} A \\
B_{t}+v_{g} B_{x}= & \mathrm{i} \alpha B_{x x}-(\delta-\mathrm{id}) B+\mathrm{i}\left(\left.\alpha_{3}\left|B^{2}-\alpha_{4}\right| A\right|^{2}\right) B+\mathrm{i} \alpha_{5} \mu \bar{A} \\
& -\mathrm{i} \alpha_{6} \int_{-1}^{0} g(y)\left(w_{y}^{m}\right)^{x} \mathrm{~d} y B-\mathrm{i} \alpha_{7}\left\langle f^{m}\right)^{x} B \\
A(x+L, t) \equiv & A(x, t), \quad B(x+L, t ; \equiv B(x, t)
\end{aligned}
$$

where $\mu$ denotes the (small) amplitude of the periodic forcing. The first seven terms in these equations, accounting for inertia, propagation at the group velocity $v_{g}$, dispersion, damping, detuning: cubic nonlinearity and 


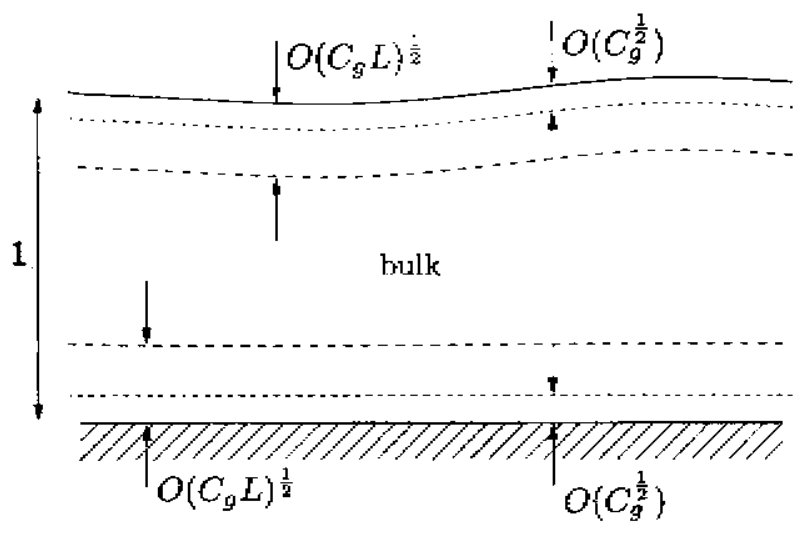

Fygure 2.2. Sketch of the primary and secondary boundary layers, indicating their widths in comparison to the layer depth.

parametric forcing, are familiar from existing weakly nonlinear, ncarly inviscid theories (Ezerskii, Rabinovich, Reutor, and Starobincts [1986]). These theories lead to expression (2.9) for the damping $\delta$ and the expressions

$$
\begin{aligned}
& v_{9}=\omega^{\prime}(k), \quad \alpha-\omega^{\prime \prime}(k) / 2, \\
& \alpha_{3}-\frac{\left.\omega k^{2}\left[(1 \quad S)\left(9-\sigma^{2}\right)(]-\sigma^{2}\right)+S k^{2}\left(7-\sigma^{2}\right)\left\langle 3-\sigma^{2}\right)\right]}{4 \sigma^{2}\left[(1-S) \sigma^{2}-S k^{2}\left(1,-\sigma^{2}\right)^{2}\right.} \\
& \quad+\frac{\left[8(1 \quad S) \cdot 5 S k^{2}\right]}{4\left(1-S+S k^{2}\right)}, \\
& \alpha_{4}=\frac{\omega k^{2}}{2}\left[\frac{\left(1-S-S k^{2}\right)\left(1-\sigma^{2}\right)^{2}}{\left(1-S-4 S k^{2}\right) \sigma^{2}}+\frac{4(1-S)+7 S k^{2}}{1-S+S k^{2}}\right], \\
& \alpha_{5}=\omega k \sigma,
\end{aligned}
$$

where $\omega=\omega(k)$ is the dispersion relation $\{2.8\}$. and are recovered in the present formulation. In particular, the cubic coefficients coincide with those obtained in strictly inviscid formulations (Pierce and Knobloch [1994]; see also Miles [1993], Hansen and Alstrom [1997] and references therein). The coefficient $\alpha_{3}$ diverges at (excluded) resonant wavenumbers satisfying $\omega(2 k)=2 \omega(k)$. The last two terms describe the coupling to the mean flow in the bulk (be it viscous or inwiscid in origin) in terms of (a local average \langle\rangle$^{x}$ of) the streamfunction $t^{\text {th }}$ for this flow and the associated free surface elevation $f^{m}$. The coefficients of these terms and the function $g$ are given 
by

$$
\alpha_{6}=\frac{k \sigma}{2 \omega}, \quad \alpha_{7}=\frac{\omega k\left(1-\sigma^{2}\right)}{2 \sigma}, \quad g(y)=\frac{2 \omega k \cosh [2 k(y+1)]}{\sinh ^{2} k} .
$$

The new terms are therefore conservative: implying that at leading order the mean flow does not extract energy from the systcm. This result is consistent with the small steepness of the associated surface displacement and its small speed compared with the speed $\nabla \psi \mid$ due to the surface waves. The mean flow variables in the bulk depend weakly on time but strongly on both $x$ and $y$, and evolve according to the equations

$$
\begin{aligned}
& \psi_{x x}^{m}+\psi_{y y}^{m}=\Omega^{m}, \\
& \Omega_{t}^{m}-\left\{\psi_{y}^{m}+(\mid A\}^{2}-|B|^{2}\right) g(y) \Omega_{x}^{m}-\psi_{x}^{m} \Omega_{y}^{m}=C_{g}\left(\Omega_{x x}^{m}+\Omega_{y y}^{m}\right), \\
& \psi_{x}^{m}-f_{t}^{m}=\beta_{1}\left(|B|^{2}-|A|^{2}\right\rangle_{x}, \quad \psi_{y y}^{m}=\beta_{2}\left(|A|^{2}-|B|^{2}\right), \quad \text { at } y=0, \\
& \begin{aligned}
(1-S) f_{x}^{m}-S f_{x x x}^{m}-\psi_{y t}^{m}+C_{g}\left(\psi_{y y y}^{m}-3 \psi_{x x y}^{m}\right) & \\
& =-B_{3}\left\{|A|^{2}+\mid B^{2}\right\}_{x}, \quad \text { at } y=0,
\end{aligned} \\
& \int_{0}^{L} \Omega_{y}^{m} d x=\dot{\psi}^{m}=0, \quad \text { at } y=-1, \\
& \left.\psi_{y}^{m}=-\left.\beta_{4}\left|\mathrm{i} A \bar{B} e^{2 i k x}+c_{0}+\right| S\right|^{2}-|A|^{2}\right], \quad \text { at } y--1, \\
& \psi^{m}(x+L, y, t)=w^{m}(x, y, t), \quad f^{m}(r-L, t)=f^{m}(x, t),
\end{aligned}
$$

subject to the constraint.

$$
\int_{0}^{p} f^{m}(x, t) d x=0
$$

Here

$$
\begin{array}{ll}
\beta_{1}=2 \omega / \sigma, & \beta_{2}=8 \omega k^{2} / \sigma \\
\beta_{3}=\left(1-\sigma^{2}\right) \omega^{2} / \sigma^{2}, & \beta_{4}=3\left(1-\sigma^{2}\right) \omega k / \sigma^{2} .
\end{array}
$$

Thus the mean fow is forced by the surface waves in two ways. The right sides of the boundary conditions (2.19a) and (2.20) provide a normal forcing mechanism; this mechanism is the only one present in strictly inviscid thcory (Davey and Stewartson 1974]: Pierce and Knobloch [1994]) and does not appear unless the aspect ratio is large. The right sides of the boundary conditions (2.19b) and (2.21c) describe two sheor forcing mechanisms, a 
tangential stress at the frte surface and a tangential velocity at the bottom wall. Note that, as in the simpler example considered in $\$ 1$, neither of these forcing terms vanishes in the limit of small viscosity (i.e., as $C_{g} \rightarrow 0$ ). The shear nature of these forcing terms leads us to retain the viscous term in (2.18b) even when $C_{3}$ is quite small. In fact, when $C_{g}$ is very small, the effective Reynolds number of the mean flow is quite large. Thus the mean flow itself generates additional boundary layers near the top and bottom of the containcr, and these must be thicker than the original boundary layers for the validity of the analysis. This puts an additional restriction on the validity of the equations (Vega. Knobloch, and Martel (2001]). There is a third, less effective but inviscid, volumetric forcing mechanism associated with the sccond term in the vorticity equation $(2.18 \mathrm{~b})$, which looks like a horizontal force $\left(\left.\left|A^{2}-\right| B\right|^{2}\right) g(y) \Omega^{m}$ and is sometimes called the vortex force. This term plays an important role in the generation of Langmuir circulation (Lcibovich 1983)). A:though this term vanishes in the absence of mean flow, it can change the stability properties of the flow and enhance or limit the effect of the remaining forcing terms. However, this is not the case in the limit considered in $\$ 3$ below

In the following we refer to Eqs. (2.11)-(2.13) and $(2.18)-(2.23)$ as the general coupled amplitude-mean-flow (GCAMF) equations. These equations differ from the exact equations forming the starting point for the analysis in the presence of the forcing terms in the boundary conditions (2.19)-(2.21), and in two essential simplifications: the fast oscillations associated with the surface waves have been fittered out, and the boundary conditions are applied at the unperturbed location of the free surface, $y=0$. The forcing terms capture completely the effect of the primary viscous boundary layers on the bulk.

The GCAMF equations are invariant under reflection.

$$
\psi^{m} \rightarrow-\psi^{m}, \quad \Omega^{m} \rightarrow-\Omega^{i n}, \quad A \leftrightarrow B, \quad x \rightarrow-x,
$$

and hence admit reflection-5ymmetric snititions. The simplest such solutions are the spatjally uniform standing waves given by $A=B=R \mathrm{e}^{\mathrm{i} \theta}$, where $\theta$ is a constant and the amplitude $R$ is given by

$$
\delta^{2}+\left[a+\left(\alpha_{3}-\alpha_{4}\right) R^{2-2}=a_{5}^{2} \mu^{2},\right.
$$

with an associated reflection-symmetric streaming flow that is periodic in $x$ with period $\pi / k$ (see Eq. (2.21ci). Since this mean flow does not couple to the amplitudes $A, B$ (i.e., the mean fow terms are absent from Eqs. (2.11)(2.12)), the presence of this flow does not affect the standing waves. These much-studied waves bifurcate from the lat state at

$$
\mu=\mu_{c} \equiv \frac{\left(\delta^{2}+d^{2}\right)^{-/ 2}}{\alpha_{5} \mid},
$$

and do so supercritically if $d<0$ and subcritically if $d>0$, see Fig. 2.3. Note that $\mu$ can be of order $\mu_{c}$ without violating the conditions for the validity of 
the GCAMF equations, and that these equations describe correctly both cases $d<0$ and $d>0$. In the former case, the waves are stable near threshold, but may lose stability at finite amplitude through the action of the mean flow as the forcing amplitude increases. Like the secondary saddlenode bifurcation which stabilizes the spatially uniform standing waves when $d>0$ (see Fig. 2.3), this bifurcation is well within the regime of validity of the GCAMF equations. Thus the mearl fow is involved only in possible secondary instabilities of the primary standing wave branch.

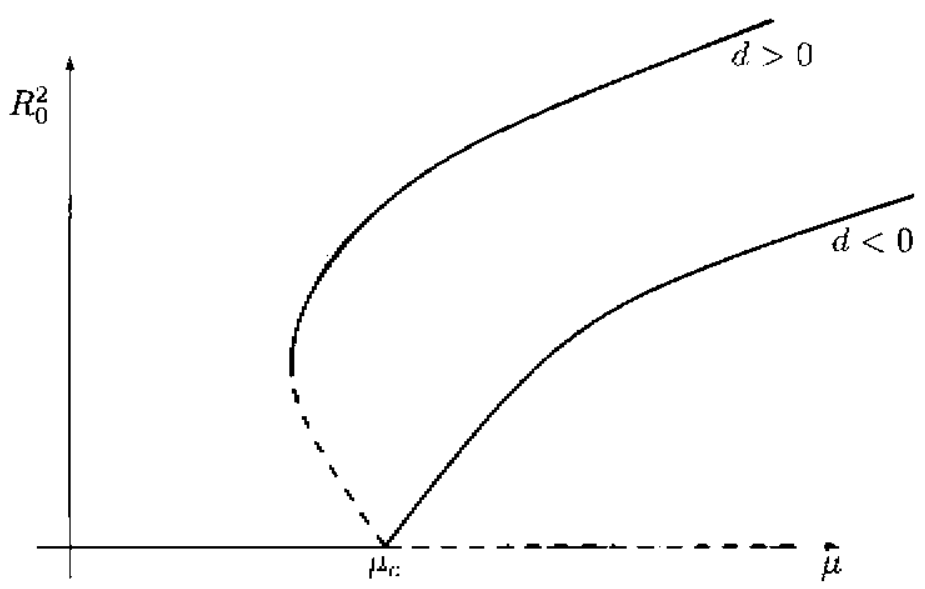

VICURE 2.3. The primary bifurcation from the fiet state to spalially unjorm standing wave solutions. The GCAMIF equations describe correctly all states with $\left|\mu-\mu_{r}\right| \sim \mu_{r}$, including the sooondary saddle node bifurcation present when $d>0$ and the stathlo solutions tryorst it.

The special case $d=0$ (zero detuning) and $s_{b}=\mu_{c}$ defines a codimensiontwo point for the analysis since both $L$ (or equivalcntly $w$ ) and $\mu$ must be chosen appropriately. In this case the direction of branching is determined by higher order terms neglected in the analysis, such as the real parts of the coefficients of the cubic terms, and this is so for sufficiently small but nonzero values of $d$ as well. In other words, the limit $a \rightarrow 0$ (although welldefined within the GCAMF equations) may not describe correctly the corresponding behavior of the underlying fluid equations appropriately close to threshold, i.e., for $\left|\mu-\mu_{c}\right| \ll \mu_{c}$. However, even in this casc the GCAMF equations capture correctly any secondary instabilities involving the mean flow, provided these occur at $\mu \sim \mu_{r}$. A similar remark applies to other codimension-t.wo points as well. 


\section{Gravity-Capillary Waves in Moderately Large Aspect-Ratio Containers}

The GCAMF equations deseribe small amplitude slowly varying wavetrains whenever the parameters $C_{g}, L^{-1}$ and $\mu$ are small, but otherwise unrelated to one another. Any relation between them wist therefore lead to further simplification. To derive such simplified equations we consider the distinguished limit

$$
\delta L^{2} / \alpha=\Delta \sim 1, \quad d L^{2} / \alpha=D \sim 1, \quad \mu L^{2} / \alpha \equiv M \sim 1,
$$

with $1 \lesssim k \lesssim\left|\ln C_{g}\right|$, and $\left|\ln C_{g}\right|$ taken for simplicity to be $O(1)$ as well. The simplified equations will then be formally walid for $1 \ll L \ll C_{g}^{-1 / 2}$ if $k \sim 1$. These are derived under the assumption $1-S \sim 1$ using a multiple scale method with $x$ and $t$ as fast wariables and

$$
\zeta=x / L, \quad \tau=t / L, \quad T=t / L^{2}
$$

as slow variables. In terms of these variables the local horizontal average $\langle\cdot\rangle^{x}$ becomes an average over the fast variable $x$. Note that assumption (3.1) imposes an implicit relation between $L$ and $C_{g}$. When $1-S \sim 1$ the nearly inviscid and viscous mean flows can be clearly distinguished from one another as diseussed in $\$ 2$, and the viscous mean flow can be identified by taking appropriate averages of the whole mean flow over an intermediate timescale $T$, i.e., the mean flow variablos $\eta^{m}, s^{m}$ and $f^{m}$ take the form

$$
\begin{aligned}
\psi^{m}(x, y, \zeta ; \tau, T) & =\psi^{v}(x, y, \zeta, T)+\psi^{i}(x, y, \zeta, \tau, T), \\
\Omega^{m}(x, y, \zeta, \tau, T) & =\Omega^{\prime \prime}(y, y, \zeta, T)+\Omega^{i}(x, y, \zeta, \tau, T), \\
f^{m}(x, \zeta, r, T) & =f^{\prime \prime}(x, \zeta, T)+f^{\prime \prime}(x, \zeta, T, T),
\end{aligned}
$$

with

$$
\left|\int_{0}^{\tau} \psi_{x}^{2} d \tau\right|+\left|\int_{0}^{\tau} \psi_{\zeta}^{i} d \tau\right|-\left|\int_{0}^{\tau} \psi_{y}^{i} d \tau\right|-\left|\int_{0}^{\tau} \Omega^{i} d \tau\right|+\left|\int_{0}^{\tau} f^{i} d \tau\right|
$$

bounded as $\tau \rightarrow \infty$. Thus the nearly inviscid mean flow is purely oscillatory (i.e., it has a zero mean) on the timescale $\tau$. Since its frequency is of the order of $L^{-1}$ (see (3.2)), which is large compared with $C_{g}$, the inertial term for this flow is large in comparison with the viscous terms (see Eq. (2.18)), except in two secondary boundary layers, of thickness of the order of $\left(C_{b} L\right)^{1 / 2}(\ll 1)$, attached to the bottom plate and the free surface. Note that, as required for the consistency of the analysis, these boundary layers are much thicker than the primary boundary layers associated with the surface waves (see Fig. 2): which provide the boundary conditions (2.19)-(2.21) for the mean flow. Horeovcr, the width of these secondary 
boundary layers remains small as $\tau \rightarrow \infty$ and (to leading order) the vorticity of this nearly inviscid mean flow remains confined to these boundary layers. This is because, according to condition (3.6), the nearly inviscid mean flow is purely ascillatory on the timescale $\tau$. Consequently, condition (3.6) is essential for the validity of the analysis that follows, and the mathematical definition of the nearly inviscid mean flow through Eqs. (3.3)-(3.6) is the only consistent one; without this condition vorticity would diffuse outside the boundary layers and affect the structure of the whole 'nearly inviscid' solution even at leading order. In fact, vorticity does diffuse (and is convected) from the boundary layers, but this vorticity transport is included in the viscous mean flow. The vorticity associated with the ncarly inviscid mean flow is readily seet to be o:, at most, the order of

$$
\left.|| A\right|^{2}-|B|^{2} \mid \text { and }\left(|A|^{2}+B^{2}\right)\left(C_{g} L\right)^{-1 / 2}
$$

in the upper and lower secondary boundary layers, respectively; the jump in the associated streamfunction $w^{i}$ across each boundary layer is $O\left(C_{g} L\right)$ times smaller. This jump only affects higher order terms; as a consequence the secondary boundary layers can be completely ignored and no additional contributions to the boundary conditions on the nearly inviscid flow need be included in (2.19) and (2.21). Outside these boundary layers, the complex amplitudes and the flow variables associated with the nearly inviscid mean flow are expanded as

$$
\begin{aligned}
(A, B) & =L^{-1}\left(A_{0}, B_{0}\right)+L^{-2}\left(A_{1}, B_{1}\right)+\cdots, \\
\left(\psi^{i}, f^{i}, \Omega^{\prime}\right) & =L^{-2}\left(\phi_{0}^{i}, F_{0}^{i}, 0\right)+L^{-3}\left(\bigcup_{1}^{i}, F_{1}^{i}, W_{0}^{i}\right)+\cdots, \\
\left(\psi^{v}, f^{v}, \Omega^{v}\right) & =L^{-2}\left(\phi_{0}^{i}, 0, W_{0}^{v}\right) \cdot L^{-3}\left(\phi_{1}^{i}, F_{0}^{v}, W_{1}^{v}\right)+\cdots .
\end{aligned}
$$

Substitution of (3.1) (3.6) $(3.8,-(3.10)$ into $(2.11)(2.23)$ leads to the forlowings:

(i) From (2.18) (2.21), at leadiug order, $\phi_{0 x x}^{i}+\phi_{0 y y}^{i}=0$ in $-1<y<0, \quad \phi_{0}^{i}=0$ at $y=-1, \quad \phi_{0 x}^{i}=0$ at $y=0$, together with $F_{0: \mathrm{r}}^{i}=0$. Thus

$$
\phi_{0}^{i}=(y+1) \Phi_{0}^{i}(\zeta, \tau, T): \quad F_{\mathrm{C}}^{i}=F_{0}^{i}(\zeta, \tau, T) .
$$

At second order, the boundary conditions (2.19a) and (2.20) yield

$$
\begin{aligned}
\phi_{1 x}^{2}(x, 0, \zeta, \tau, T) & \left.=F_{0 \tau}^{i}-\Phi_{0 \zeta}^{i}+\left.\beta_{1(i,} B_{0}\right|^{2}-\left|A_{0}\right|^{2}\right)_{\zeta}, \\
(1-S) F_{1 x}^{i}-S F_{1 x: x x}^{i} & =\Phi_{0 \tau}^{i}-(1-S) F_{0 \zeta}^{i}-\beta_{3}\left(\left|A_{0}\right|^{2}+\left|B_{0}\right|^{2}\right)_{\zeta}
\end{aligned}
$$

at $y=0$. Since the right hand sides of these two equations are independent of the fast variable $x$ and both $\phi_{1}^{i}$ and $F_{1}^{i}$ must be bounded in $x$, it follows 
that

$$
\begin{aligned}
\Phi_{0 \zeta}^{i}-F_{0 \tau}^{i} & =\beta_{1}\left(\left|B_{0}{ }^{2}-A_{0}\right|^{2}\right)_{\zeta}, \\
\Phi_{0 \tau}^{i}-v_{\mathrm{p}}^{2} F_{0 \zeta}^{i} & =\beta_{3}\left(\left|A_{0}\right|^{2}+\left|B_{0}\right|^{2}\right)_{\zeta},
\end{aligned}
$$

where

$$
v_{p}=(1-S)^{1 / 2}
$$

is the phase velocity of long wavelength surface gravity waves. Equations (3.12) must be integrated with the following additional conditions, which result from $(2.22)-(2.23)$ and $(3.6)$.

$$
\begin{gathered}
\Phi_{0}^{i}(\zeta+1, \tau, T) \equiv \Phi_{0}^{i}(\zeta: \tau, T): \quad F_{0}^{i}(\zeta+1, \tau, T) \equiv F_{0}^{i}(\zeta, \tau, T), \\
\qquad \int_{0}^{1} F_{0}^{i} d \zeta=0, \\
\left|\int_{0}^{\tau} \Phi_{0 \zeta}^{i} d \tau\right|+\left|\int_{0}^{\tau} F_{0}^{i} d \tau\right|=\text { bounded as } \tau \rightarrow \infty .
\end{gathered}
$$

(ii) The leading order contributions to equations (2.11)-(2.12) yield

$$
A_{0 \mathrm{~T}}-v_{\mathrm{s}} A_{0 \mathrm{k}}=B_{0 \mathrm{r}}+v_{\mathrm{g}} B_{0 \mathrm{C}}=0
$$

Thus

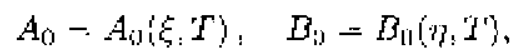

where $\xi$ and $\eta$ are the characlerjstic variables

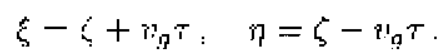

Moreover, according to $(2.13)$,

$$
A_{0}(\xi+1, T) \equiv A_{0}(\xi, T), \quad B_{0}(\eta+1, T) \equiv B_{0}(\eta, T)
$$

Substitution of these expressions into (3.12) followed by integration of the resulting equations yields

$$
\begin{aligned}
& \Phi_{0}^{i}=\frac{\beta_{3} v_{p}^{2}+\beta_{3} v_{g}}{v_{g}^{2}-v_{p}^{2}}\left[\left.{ }^{2} A_{0}\right|^{2}-\left|B_{0}\right|^{2}-\left\langle\left|A_{0}\right|^{2}-\left|B_{0}\right|^{2}\right\rangle \zeta\right] \\
& +v_{p}\left[F^{+}\left(\zeta+v_{p} T, T\right)-F^{-}\left(\zeta-v_{p} \tau, T\right)\right] \text {, } \\
& \left.F_{0}^{i}=\frac{\beta_{1} v_{g}+\beta_{3}}{v_{g}^{2}-v_{\mathrm{p}}^{2}}\left|A_{0}\right|^{2}-\mid B_{0}{ }^{2}-\left(\left|A_{0}\right|^{2}+\left|B_{0}\right|^{2}\right\rangle^{\zeta}\right] \\
& +\left[F^{\frac{1}{2}}\left(\zeta+v_{p} \tau: T\right)+F^{-}\left(\zeta-i_{p} T, T\right)\right],
\end{aligned}
$$


where $(\cdot\rangle^{\varsigma}$ denotes the mean value in the slow spatial variable $\zeta$, i.e.,

$$
(G) \zeta=\int_{0}^{1} G d \zeta
$$

and the functions $F^{\text {上 }}$ are such that

$$
\left.F^{ \pm}\left(\zeta+1 \pm v_{p} T, T\right) \equiv F^{ \pm} \zeta \pm \nu_{p} T, T\right), \quad(F=) \zeta=0
$$

The particular solution of (3.19)-\{3.20) yields the usual inviscid mean flow included in rearly inviscid theories (see Pierce and Knobloch [1994] and references therein ; the averaged terms are a consequence of the conditions (3.15), i.e., of volume conserwation (cf. Pierce and Knobloch [1994]) and the requirement that the rearly intiscid mean flow has a zero mean on the timescale $\tau$; the latter condition is never imposed in strictly inviscid theories but is essential in the limit we are considering: as explained above. To avoid the breakdown of tine solution $(3.19)-(3.20)$ at $v_{p}=v_{g}$ we assume that

$$
\left|\tau_{p}-\tau_{g}\right| \sim 1 \text {. }
$$

The functions $F^{ \pm}$remain undetermined at this stage. In fact, they are not needed below because the evolution of both the viscous mean flow and the complex amplitudies is decoupled from these functions. However, at next order one finds that $F=$ remain constant on the timescale $T$, but decay exponentially due to viscous offects (resulting from viscous dissipation in the secondary bomdary layer attached to the bottom plates on the timescale $t \sim\left(L / C_{y}\right)^{1 / 2}$.

(iii) The evolution equations for $A_{1 \mathrm{l}}$ and $B_{0}$ on the timescale $T$ are readily obtained from cquations (2.11)-(2.13), invoking (3.1)-(3.6), (3.19)-(3.20), (3.22) ard olininating senular terms (i.e., requiring $\left|A_{1}\right|$ arrel $\left|D_{1}\right|$ to be bounded on the timcsicale 1 ;:

$$
\begin{aligned}
& A_{0 T}=\mathrm{i} \alpha A_{0 \xi \xi}-(\mathrm{A}-\mathrm{i} D) A_{0} \\
& \left.+\mathrm{i}\left[\left.\left(\alpha_{3}+\alpha_{8}\right) \cdot A_{0}\right|^{2}-\left.\alpha_{8}|| A_{0}\right|^{2}\right\rangle^{\xi}-\alpha_{4}\left\langle\left|B_{0}\right|^{2}\right\rangle^{\eta}\right] A_{0} \\
& +\mathrm{i} \alpha_{5} M\left(\bar{B}_{0}\right)^{n}+\mathrm{i} \alpha_{6} \int_{-1}^{0} g(y)\left(\left(\phi_{0 y}^{2}\right)^{x}\right)^{\zeta} d y A_{0} \text {, } \\
& B_{0 T}=\mathrm{i} \alpha B_{0 \eta \eta}-(\mathrm{A}+\mathrm{i} D) B_{0} \\
& \left.+\mathrm{i}\left[\left.\left(\alpha_{3}+\alpha_{8}\right)^{\prime} B_{0}\right|^{2}-\alpha_{8}\langle| B_{0}^{2}\right)^{\eta}-\alpha_{4}\left(\left|A_{0}\right|^{2}\right)^{\xi}\right] B_{0} \\
& +\mathrm{i} \alpha_{5} M\left(\bar{A}_{0}\right)^{\xi}-\mathrm{i} \alpha_{6} \int_{-1}^{\mathrm{n}} g(y)\left(\left\langle\phi_{05}^{2}\right)^{x}\right)^{\varsigma} d y B_{0} \text {, }
\end{aligned}
$$

subject to (3.18). Here $\xi$ and $\eta$ are the comoving variables defined in (3.17), and $\langle\cdot\rangle^{x},\langle\cdot\rangle^{\zeta},(\cdot\rangle^{\xi}$ and $(\cdot\rangle^{\eta}$ denote mean values over the variables $x, \zeta, \xi$ and $\eta$, respectively. Note that $\zeta$ averages over functions of $A_{0}$ are equivalent 
to $\xi$ averages, while those ower functions of $B_{3}$ are equivalent to $\eta$ averages. The real coefficient $\alpha_{8}$ is given by

$$
\alpha_{8}=\left[\alpha_{s}(2 \omega / \sigma)\left(\beta_{1} \tau_{p}^{2}+\beta_{3} v_{g}\right)+\alpha_{7}\left(\beta_{1} v_{37}+\beta_{3}\right)\right] /\left(v_{g}^{2}-v_{p}^{2}\right)
$$

Eqs. (3.24)-(3.25) are independent of $F^{ \pm}$because of the second condition in $(3.22)$.

Since

$$
\left\langle\left|A_{0}\right|^{2}-\left|B_{0}\right|^{2}\right\rangle^{\top}=\left(\left.A_{0}\right|^{2}\right\rangle^{c}-\left(\mid B_{0}^{2}\right)^{n} \rightarrow 0 \text { as } T \rightarrow \infty
$$

the long time behavior of the viscous mean flow is described by

$$
\begin{aligned}
& \phi_{0 x x}^{v}+\phi_{0 y y}^{v}=W_{0}^{n} \text { in }-1<y<0 \text {. } \\
& W_{0 T}^{v}-\phi_{0 y}^{v} W_{0 x}^{v}+\phi_{0 x}^{v} W_{0 y}^{v}=R e^{-1}\left(W_{0 x x}^{v}+W_{0 y y}^{v}\right) \quad \text { in }-1<y<0 \text {, } \\
& \phi_{0 x}^{v}=\phi_{0 y y}^{v}=0 \text { at } y=0 \text {. } \\
& \left\langle\left\langle W_{0 y}^{v}\right\rangle^{x}\right\rangle^{\varsigma}=\phi_{0}^{v}=0 \text { at } y=-1 \text { : } \\
& \phi_{0 y}^{v}=-\beta_{4}\left[\mathrm{i}\left\langle A_{0} \bar{B}_{0}\right\rangle^{\top} \mathrm{e}^{2 \mathrm{i} \dot{k} x}+\text { c.c. }\right] \text { at. } y=-1 \text {, } \\
& \phi_{0}^{v}(x+L, \zeta+1, y, T) \equiv \phi_{0}^{v}(x, \zeta: y, T)
\end{aligned}
$$

where the effective Reynolds number associated with this viscous mean flow is

$$
R e=1 /\left(C_{0} I^{2}\right)
$$

Remarks. Some remarks abuut these equations and boundary conditions are now in order.

a. The viscous menn fow is driven by the short gravity-capillary waves through the inhowogeneous term in the boundary condition (33.31). Since $\left\langle A_{0} B_{0}\right\rangle^{\tau}$ depends on both 5 and $T$ (tunless either $A_{0}$ or $B_{0}$ is spatially uniform) the boundary condition implies that $\phi_{0}^{v}$ (and hence $W_{0}^{v}$ ) depends on both the fast and slow horizontal spatial variables $x$ and $\zeta$. This dependence cannot be obtained in closed form (except, of course, in the uninteresting limit $R e \rightarrow 0$ ), and one must resort to numerical computations for realistically large values of $L$.

b. The higher order oscillatory terms absent from the boundary condition (3.31) oscillate on the intermediate timescale $\tau$, and hence generate secondary boundary layers. However, the contributions from these boundary layers are all subdominant and have no effect at the order considered. Moreover, the free-surface deflection accompanving the viscous mean flow is also small, $f^{t t} \sim L^{-3}$ (sce Eq. (3.10)), and so plays no role in the evolution of this flow, as cxpected of a flow involving the excitation of viscous modes (see $§ 2$ ). 
c. The dominant forcing of the viscous mean flow comes from the lower boundary. This forcing vanishes exponentiatly when $k \gg 1$ leaving only a narrow range of wavenumbers within which such a mean flow is forced while $\delta=O\left(C_{g}\right)$, see Fig. 2.1. Thus in most cases in which a viscous mean flow is present one may assume that

$$
\bar{j}=O\left(C_{3}^{1 / 2}\right) .
$$

Note, however, that in fully three-dimensional situations (such as that in Douady, Fauve, and Thual [1989]\} in which lateral walls are included a viscous mean flow will be present even when $k \gg 1$ because the forcing of the mean flow in the oscillatory boundary layers along the lateral walls remains.

d. According to the scaling (3.1) and the definitions (2.8), (2.9) and (3.33), the effective Reynolds number $R e$ is large, and ranges from logarithmically large values if $k \sim \mid \ln C_{z}$ to $O\left(C_{g}^{-1 / 2}\right)$ if $k \sim 1$. However, even in the latter limit we must retain the viscous terms in $\{3.28\}$ in order to account for the second boundary conditions in (3.29) ) (3.31). Of course, if $R e \gg 1$ vorticity diffusion is likely to be confined to thin layers, but the structure and location of all these layers cannot be anticipated in any obvious way, and one must again rely on numerical computations.

e. Note that the change of variables

$$
A_{0}=\tilde{A}_{0} \mathrm{e}^{-\mathrm{i} \theta}: \quad B_{0}=\tilde{B}_{0} \mathrm{c}^{\mathrm{i} \theta} \text { : }
$$

where

$$
\theta^{\prime}(T)=-\alpha_{\theta} \int_{-1}^{m} g(y)\left\langle\left\langle\phi_{0 y}^{v}\right\rangle^{n}\right\rangle^{c} d y
$$

reduces Eqs: (3.24) (3.25) to the much simpler form

$$
\begin{aligned}
& \bar{A}_{0 T}=\mathrm{i} \alpha \bar{A}_{0 \xi \xi}-(\Delta+\mathrm{i} D\rangle \bar{A}_{0}+\mathrm{i} \alpha_{5} M\left\langle\ddot{\bar{B}}_{0}\right)^{t_{1}} \\
& +\mathrm{i}\left[\left(\alpha_{3}+\alpha_{B}\right) \mid \tilde{A}_{0}{ }^{2}-\left(\alpha_{4}+\alpha_{8}\right)\left(\left|\tilde{A}_{0}\right|^{2}\right)^{\xi}\right] \tilde{A}_{0} \text {, }
\end{aligned}
$$

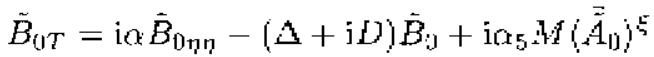

$$
\begin{aligned}
& +\mathbf{i}\left[\left(\alpha_{3}+\alpha_{5}\right)\left|\tilde{B}_{0}\right|^{2}-\left(\alpha_{4}+\alpha_{8}\right)\left\langle\left|\tilde{B}_{0}\right|^{2}\right\rangle^{n}\right] \tilde{B}_{0}, \\
& \tilde{A}_{0}(\xi+1, T) \equiv \tilde{A}_{0}(\xi, T), \quad \tilde{B}_{0}(\eta-1, T) \equiv \tilde{B}_{0}(\eta, T) \text {. }
\end{aligned}
$$

from which the mean flow is absent. This decoupling is a special property of the regime defined by Eq. (3.1). The resulting equations provide perhaps the simplest description of the Faraday system at large aspect ratio, and it is for this reason that they have been extensively studied (Martel, Knobloch, and Vega $[2000]$ ). We summarize some of their properties in the next section. 


\section{Dynamics of the Reduced Equations}

In this section we describe some basic properties of the nonlocal equations $(3.36)-(3.38)$ in the invariant subspace $\tilde{A}_{0}(\cdot$, tau $)=\tilde{B}_{0}(\cdot$, tau $) \equiv$ $C(\cdot, t a u)$, say, in which the dynamics are described by the partial differential equation (PDE)

$$
\begin{aligned}
C_{\tau}= & \mathrm{i} \alpha C_{x x}-(\Delta+\mathrm{i} D) C \\
& \left.+\mathrm{i}\left[\left.\left(\alpha_{3}+\alpha_{\bar{B}}\right)\left|C^{2}-\left(\alpha_{4}+\alpha_{s}\right)\right| C\right|^{2}\right)\right] C+\mathrm{i} \alpha_{5} M\langle\bar{C}\rangle,
\end{aligned}
$$

subject to periodic boundary conditions. Henceforth the variable $x$ stands for either $\eta$ or $\xi$, depending on whether $C$ stands for $\tilde{A}_{0}$ or $\tilde{B}_{0}$. Eq. (4.1) describes standing wave solutions of Eqs. $(3.36)-\{3.38)$. It is possible to show that such standing waves (hereafter SW) are the preferred state at onset (Riecke, Crawford, and Knobloch [1988) although at larger values of the forcing amplitude such waves may become unstable with respect to perturbations transverse to this subspace (Martel, Knobloch, and Vega [2000]); if this is so the dynamics of Eqs. (3.36) (3.37) and (4.1) will differ.

After an appropriate rescaling (and taking the complex conjugate in (4.1) if $\alpha_{3}-\alpha_{4}<0$, and changing the sign of $\alpha$ and $d$ if $\alpha_{3}+\alpha_{8}<0$ ) the standing waves obey an equation of the form

$$
\begin{aligned}
& C_{\tau}=\mathrm{i} \alpha C_{x x}-(1+\mathrm{i} d) C+\mathrm{i}\left[\mid C^{2}+\langle A-1)\left\langle|C|^{2}\right\rangle\right] C+\mu\langle\bar{C}\rangle, \\
& C(x+1, \tau)=C\left(x_{\tau} \tau\right) .
\end{aligned}
$$

Thus the relative size of the nonlines terms is measured by the single parameter $\Lambda \equiv 1-\left(\alpha_{s}+\alpha_{8}\right) /\left(\alpha_{\beta}+\alpha_{i}\right)$.

The results of solving equations (3.36) (3.38) and (4.2) (4.3) for identical paraneter values are summarized in the bifurcation diagrams shown in Fig. 4.1. These are constructent by noting that exiutions (4.2)-(4.3) imply

$$
\frac{d}{d \tau}|C|_{L_{2}}^{2}=-\left.2|| C\right|_{L_{2}} ^{2}+\mu\left(\langle\bar{C})^{2}-\text { c.C. }\right)
$$

so that successive intersections of a trajectory with the hypersurface

$$
|C|_{J_{2}}^{2}=\frac{1}{2} \mu\left(\langle\bar{C})^{2}+c . c .\right)
$$

are always well defined. In fact this surface contains all the steary states, while each periodic trajectory intersects it at least twice in each period, at the turning points in $|C|_{L_{2}}$. In the bifurcation diagrams we plot successive maxima of $\|C\|_{L_{2}}$ at each walue of $\mu$ after transients have died away. In the general case $\left(\left|\tilde{A}_{0}\right| \neq\left|\tilde{B}_{0}\right|\right)$ we likewise plot the outward intersections with the hypersurface

$$
\left.|| \tilde{A}_{0}\right|_{L_{2}} ^{2}+\|\left.\tilde{B}_{0}\right|_{L_{2}} ^{2}=\mu\left(\left\langle\overline{\tilde{A}}_{0}\right)\left(\overline{\tilde{B}}_{0}\right)-\text { c.c. }\right),
$$


corresponding to maxima in $\left\|\bar{A}_{0}\right\|_{L_{2}}^{2}+\left\|\bar{B}_{0}\right\|_{L_{2}}^{2}$. Although this procedure for generating bifurcation diagrams is convenient for most purposes it suffers from the disadvantage that it is insensitive to phase trift. Thus additional diagnostics are necessary to identify such drifts, as discussed in detail in Martel, Knobloch, and Vega [2000:

In both cases the first instability produces uniform steady solutions and these subsequently lose stability in a symmetry-breaking pitchfork bifurcation, giving rise to time-independent but spatially nonuniform states. Both these bifurcations preserve the identity $\bar{A}_{\mathrm{C}}=\tilde{B}_{0}$ and hence are common to both sets of equations. Both also preserve the spatial reflection symmetry $\mathcal{R}: x \rightarrow-x$. In the case shomn in Fjgs. 4.1a b the resulting nonuniform but reflection-symmetric states subsequently undergo a Hopf bifurcation and produce a branch of oscillatory solutions. Shortly thereafter chaos sets in, interspersed with nonuniform temporally periodic motion. Some of the observed transitions are the result of crises while others appear to be due to period-doubling cascades. Observe that the details of this behavior differ in the two figures, indicating that the imwariant subspace $\tilde{A}_{0}=\bar{B}_{0}$ does not remain attracting for all values of $\mu$. In the following we restrict attention to the origin of this more complicated dymamical behavior in Eqs. (4.2)-(4.3).

Analysis of the system (4.2)-(4.3) is complicated by the absence of wavenumber-dependent dissipation: the damping is identical for all modes. As a result the theorem of Duan, $\mathrm{Ly}$, and Titi [1996] establishing the existence of a finite-dimensional inertial manifold for a nonlacal Ginzburg-Landau of the same type and with the same boundary conditions does not apply. Nonetheless, for the wenkly damped norlinear Schrödinger (NLS) equation with direct external Eoccing, Ghidaglin 1988 was able to demonstrate the cxistence of a weak finite-rimensional attractor. "This result was improved upon by Warg [1995] who used an entergy equation to obtain strong convergence, showing that the altractor is in fact a strons, finite-dimensional, global attractor. Subsequent work (ste, n.g.. Goubet [1996]; Oliver and 'Titi [1998]) has dealt with the task of prowing suldicioual regularity proporties of the attractor. In particular, Oliver and 'Iiti :1998j showod that the global attractor for the weakly damped driwen (but. local) NLS equation with direct forcing is analytic, indicating that the Fourier expansion of a solution on the attractor converges exponentially fast (as the number of terms is increased) to the exact solution. Whe bolieve that these properties continue to hold for the nonlocal equation with parametric forcing, and explain why a simple two-mode truncation of the PDE discussed next describes the PDE dynamics so well over a large range of parameter values.

\subsection{Two-Mode Model and Basic Solutions}

In view of the fact that botin the primary and secondary bifurcations preserve the reflection symmetry $\mathcal{R}: x \rightarrow-x$ we focus on the class of 

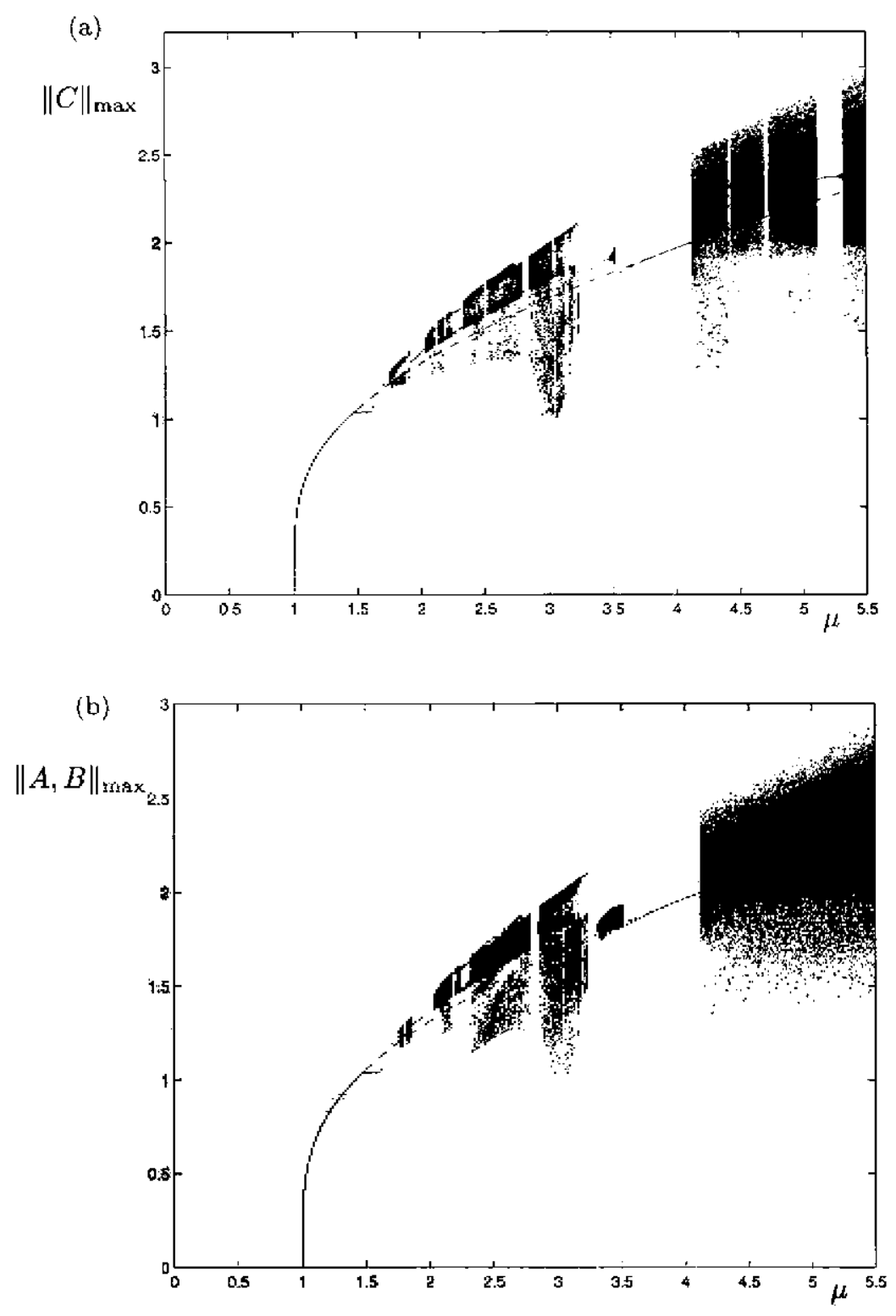

Figure 4.1. Bifurcation diagrams for the two systems described in (3.36)- (3.38) and (4.2)-(4.3), with $\alpha=0.1, d=0$ and $A=2 / 3$. Courtesy C. Martel. 
reflection-invariant states of the form

$$
C(x, \tau)=\frac{1}{\sqrt{2}} c_{0}(\tau)-\sum_{n=1}^{\infty} c_{n}(\tau) \cos (2 \pi n x) .
$$

Projection of equation (4.2) onto the first two modes then leads to the dynamical system (Hignera, Porter, and Knobloch [2002])

$$
\begin{aligned}
& \dot{c}_{0}=-(1+i d) c_{0}+i \Lambda\left(\left|c_{0}\right|^{2}-\left|c_{1}\right|^{2}\right) \frac{c_{0}}{2}+i\left(\bar{c}_{0} c_{1}-\bar{c}_{1} c_{0}\right) \frac{c_{1}}{2}+\mu \vec{c}_{0}, \\
& \dot{c}_{1}=-\left(1+i d_{1}\right) c_{1}+i \Lambda\left(\left|c_{0}\right|^{2}+\left|c_{1}\right|^{2}\right) \frac{c_{1}}{2}+i\left(\bar{c}_{0} c_{1}+\bar{c}_{1} c_{0}\right) \frac{c_{0}}{2}+i\left|c_{1}\right|^{2} \frac{c_{1}}{4},
\end{aligned}
$$

where $d_{1}=d+4 \pi^{2} \alpha$. These equations arc equivariant under the operations

$$
R_{0}:\left(c_{0}, c_{1}\right) \rightarrow\left(-c_{0}, c_{1}\right), \quad R_{1}:\left(c_{0}, c_{1}\right) \rightarrow\left(c_{0},-c_{1}\right),
$$

where $R_{0}=\hat{\kappa} T_{1 / 2}$ and $R_{1}=T_{i ; 2}$, and $T_{1 / 2}: x \rightarrow x+\frac{1}{2}, C \rightarrow C$, and $\hat{\kappa}: C \rightarrow-C$ represent two symmetries of the original equations (4.2)(4.3). These actions generate the group $D_{2}$.

Eqs. (4.8)-(4.9) contain three types of fixed points whose properties are summarized below. In what follows we set $d=0$ (both for simplicity and for comparison with Martel, Knobloch, and Vega [2000]) and write

$$
c_{0} \equiv x_{0}+i y_{0}, \quad c_{1} \equiv x_{1}+i \mho_{1},
$$

where $x_{0}, x_{1}, y_{0}$ and $y_{1}$ are al! trat.

Trivial state (O): This solution has the full symmetry $D_{2}$. Its stability is determined by the four eigcnvalues $\pm \mu \quad 1$ and $1+i \omega$, where $\omega \equiv 4 \pi^{2} \alpha$. 'l'he first two give the growth rate of forturthetions wilhin the invariant plane $c_{1}-0$, while the cumplex conjugate pair clescribes perturbations within the invariant plane $r_{0}-0$. When $p_{\alpha}-1$ there is a supercritical pitchfork bifurcation giving rise to a branch of spatially uniform states $U$ : $c_{0} \neq 0, c_{1}=0$; note that there are no fixed points of the form $c_{0}=0$, $c_{1} \neq 0$.

Uniform steady stotes (U): These solutions take the form $c_{0} \neq 0, c_{1}=0$, where

$$
|A|\left|c_{0}\right|^{2}=2 \sqrt{\mu^{2}-1}, \quad \cos 2 \theta=1 / \mu,
$$

and $c_{0}=\left|c_{0}\right| e^{i \vartheta}$, and are invariant under $R_{1}$ but not under $R_{0}$; when necessary we distinguish between the two $R_{0}$-related branches using the notation $U_{ \pm}$(the \pm reflects the sign of the $x_{n}$ coordinate). Since $d=0$ these solutions are always stable to perturbations within the plane $c_{1}=0$ with the corresponding eigenvalues, $s$, satisfying

$$
(s+1)^{2}-\left(5-4 \mu^{2}\right)=0 \text {. }
$$


Note that these eigenvalues are complex when $\mu>\sqrt{5} / 2$. Stability with respect to the mode $c_{\mathrm{I}}$ is described by the characteristic equation

$$
(s+1)^{2}+\omega^{2}+\mu^{2}-1-\frac{2}{h}\left(\mu^{2}-1\right)-\frac{2 \omega}{\mid A}(\Lambda-1) \sqrt{\mu^{2}-1}=0 .
$$

Thus, when $s=0$, the uniform states $U$ undergo a pitchfork bifurcation which breaks the $R_{1}$ symmetry and produces time-independent nonuniform states with $n=1$ (NV). Note that because of the form of Eqs. (4.12)-(4.13) Hopf bifurcations are not possible.

Nonuniform steady states ( $N U$ ): The fixed points $N U$ have no symmetry; consequently, the $N U$ states come in quartets, related by the actions of $R_{0}$, $R_{1}$, and $R_{0} R_{1}$. Depending on the value of $A$, the $N U$ states may become unstable, with increasing $\mu$, at cither a saddlc-node or a Hopf bifurcation. If a Hopf bifurcation occurs it generates four symmetry-related periodic orbits. The fate of these and other time-dependent solutions is investigated in the following section.

\subsection{Numerical Results}

In this section we present the results of a carcful ninnerical investigation of Eqs. (4.8)-(4.9) using a combination of ALTO (Doedel, Champneys, Fairgrieve, Kuznetsov, Sandstede, and Wang [1997]) and XPPAUT (Ermentrout [2000]). In addition to the simple bifurcatjons mentioned above these equations can exhibit extremely complicated dynamics. We find that over a large range of parametres this complex isebavior is organized by a codimension-one heteroclinic connection between the uniform and trivial states, a global bifurcation which can be best understood in the context of a two-parameter stouly. We therefore set $d-0_{1} a-0.1$ and vary $\Lambda$ along with the forcing amplitude $\mu$.

Fig. 4.2 shows the important local bifurcation sets in the $(\mu, \Lambda)$ plane: the $n=1$ neutral stability surve (labeled SB) and the loci of Hopf and suddlenode (SN) bifurcations on the $N E$ branch which bifurcates from the $U$ state along the neutral curve. Fig. 4.3 shows the bifureation diagrams obtained on traversing this plase in the direction of increasing $\mu$ at several different (but fixed) values of A. Fig. 4.2 reveals the presence of two singularities. There is a degeneracy when $\Lambda=0$ : at this walue of $A$ spatially uniform states exist only at $\mu=1$ and at no other value of $\mu$. It is thus not surprising that there are many bifurcation sets emanating from the singular point $(\mu, \Lambda)=(1,0)$. In the present problem there is, in addition, evidence of singular behavior at $\Lambda \simeq-1.1428$, where the amplitude of the $N U$ branch (but not the $U$ branch) bccomes infinite. As $A$ decreases toward this value the two saddlenode bifurcations on the $N U$ branch (at $\mu \sim 2.33$ and $\mu \sim 5.67$ ) occur at roughly constant $\mu$ values but at larger and larger amplitude (see Fig. $4.3 \mathrm{~g}$ ). When $\Lambda<-1.1428$ these two saddle-node bifurcations no longer occur at all (see Fig. 4.3h). 


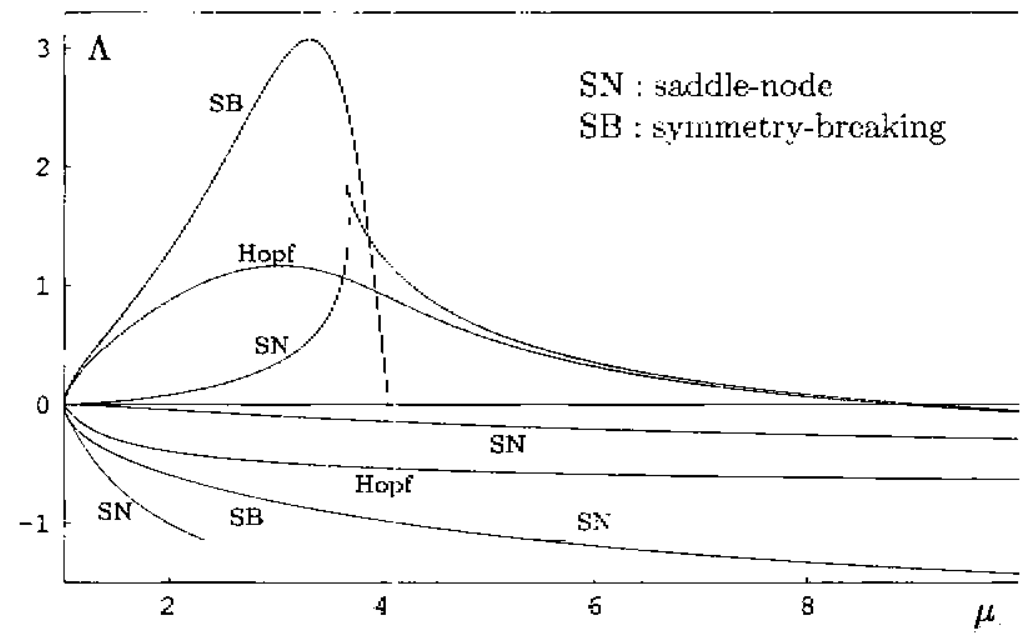

FIGURe 4.2. Local bifurcation sets with $d=0$ and $\alpha=0.1$ : symmetry-breaking bifurcation (SB) on the $U$ branch, and Hopt and saxldle-node (SN) bifurcations on the $N U$ branch. Courtesy h. Higuera and .J. Porter.

The bifurcation diagrams of Fig. 4.3 show the $U$ and $N U$ branches, as well as recording the fate of the branches of periodic orbits (when present) generated in Hopf bifurcations on the $N E$ branch (Figs. 4.3a-f). For typical parameter values the $N U$ brench is S-shaped, whth the Hopf bifurcations occurring on the lower part. For example; a cut (not shown) at $\Lambda=1$ barely crosses the locus of fIopf bifirestiosts but does so twice in quick sucression indicating the prescrice of two Hopt bifurcations back to back (see Fig. 4.2); connecting these bifurentions is a stathle brinteh of periodic: orbits. With $A=0.9$ (Fig. $4.3 \mathrm{a}$ ) there is a period-doubling bifurcation on this original brame bot the cascade (not shown) is incomplete (there are just two period-denblings followed by two reverse period-doublings). Bifurcation "bubbles" of this type are familiar from problems related to the Shil'nikov bifurcation (Knobloch and Weiss [1981; Glendinning and Sparrow [1984]). For $\Lambda=2 / 3$ (F:g.4.3b;, the walue corresponding to Fig. 4.1, there is (presumably) a complete period-doubling cascade and one can easily find a variety of periodic and chaotic attractors (see Fig. 4.4). Evidence that this cascade is not the whole story: however, is provided in Fig. 4.3c. The figure shows that, for $A=0.645$, the branch of periodic orbits has split apart, each half terminating in a Shil nikov-type homoclinic connection with the uniform state. The abruptness of this transition suggests the presence of other periodic orbits with which the original periodic branch is colliding. This interpretation is further supported by a second abrupt transition which occurs by $\Lambda=0.633$ (Fig. 4.3d): the branch of periodic states produced in the second Hopf bifurcation (at $\mu \simeq 4.8$ ) now terminates in a 

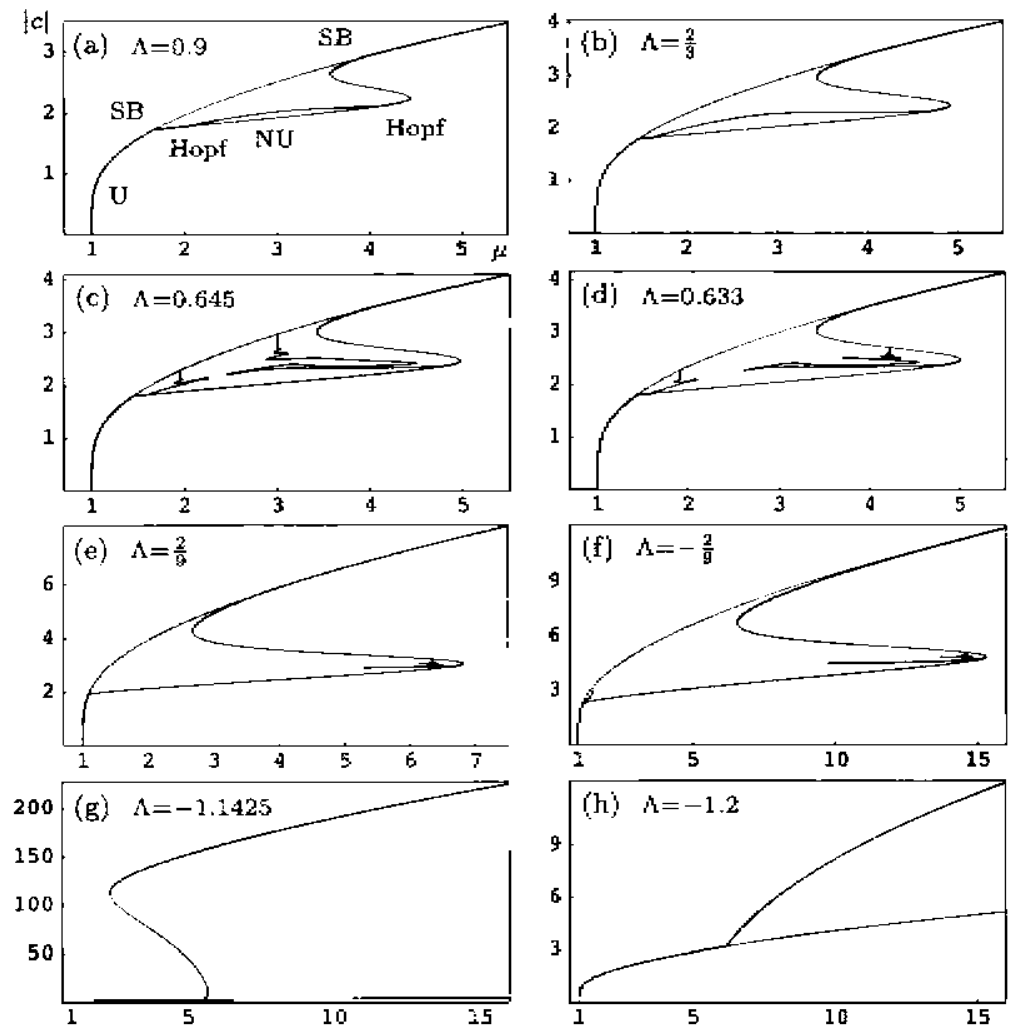

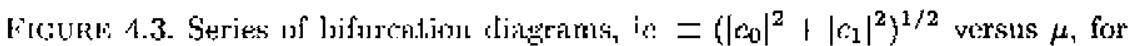
different values of $\Lambda$. Stable (unteble) bolutions are renclered with thick (thin) lines. Branches of periodic solutions originating jn Ilopf bifurcations are also shown. Courtesy M. Higuera and J. Forter.

homoclinic bifurcation on the $N U$ states rather than the $U$ states. As $\Lambda$ is decreased even further (see Fig. 4.3e) the first homocinic bifurcation (with the $U$ state) moves very close to the initial Hopf bifurcation, occurring at $\mu \simeq 1.112$ when $\Lambda=2 / 9$, while the second homoclinic bifurcation (on the $N U$ branch) moves closer to the rightmost saddle-node bifurcation. The branch of periodic solutions corresponding to the former is almost invisible on the scale of the figure. A comparison of Figs. 4.3e and 4.3f shows that when $\Lambda$ is small in magnitude the bifurcation diagrams on either side of $\Lambda=0$ are qualitatively similar. The main differences are the change in scale (larger $\mu$ values for negative $A$ ) and the absence of the rightmost symmetry-breaking (SB) bifurcation when $A<0$ : although the $N U$ branch comes very close to the $U$ branch for large $\mu$ the two branches remain 

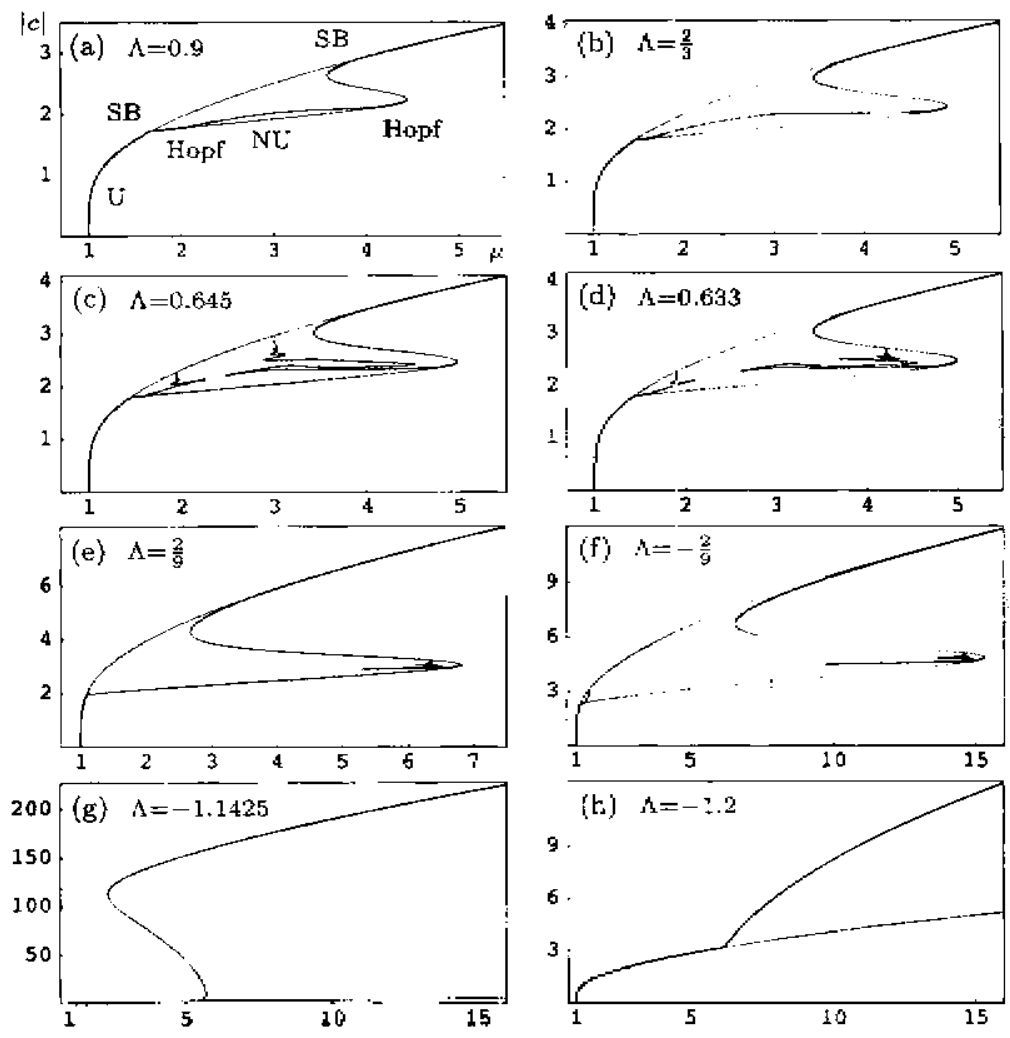

FIgURE 4.3. Series of bifurention diagratrs, $|c|=\left(\left|\mathrm{r}_{0}\right|^{2}+\left|c_{1}\right|^{2}\right)^{1 / 2}$ versus $\mu$, for different values of $A$. Stable (unstable) solutions are rendered with thick (thin) lines. Branches of periodic solutions oripinating in Hopf bifurcations are also shown. Courtesy M. Higuera and I. Forter.

homoclinic bifurcation on the $N U$ states rather than the $U$ states. As $\Lambda$ is decreased even further (see Fig. 4.3e) the first homoclinic bifurcation (with the $U$ state) moves very close to the initial Hopf bifurcation, occurring at $\mu \simeq 1.112$ when $A=2 / 9$, while the second homoclinic bifurcation (on the $N U$ branch) moves closer to the rightmost saddle-node bifurcation. The branch of periodic solutions corresponding to the former is almost invisible on the scale of the figure. A comparison of Figs. 4.3e and 4.3f shows that when $\Lambda$ is small in magnitude the bifurcation tiagrams on either side of $\Lambda=0$ are qualitatively similar. The main differences are the change in scale (larger $\mu$ values for negative $\Lambda$ ) and the absence of the rightmost symmetry-breaking (SB) bifurcation when $A<0$ : although the $N U$ branch comes very close to the $U$ branch for large $\mu$ the two branches remain 
(a)

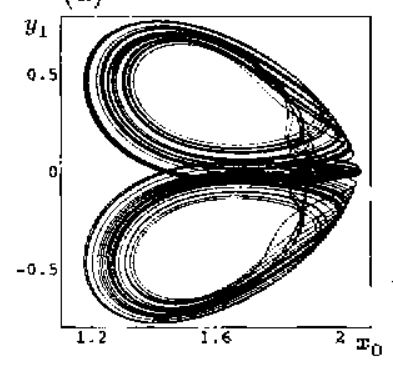

(b)

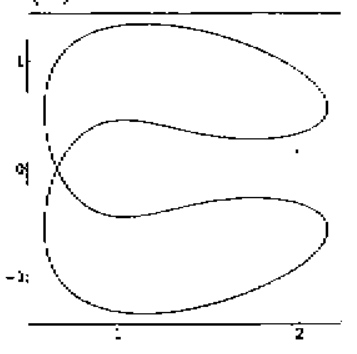

(c)

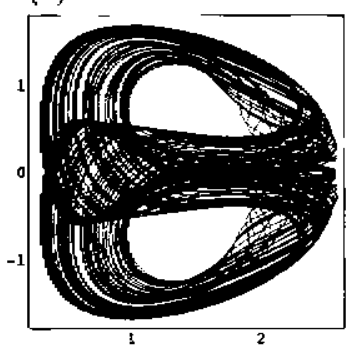

FigtiRe 4.4. Attractors for $d=0, \alpha=0.1, h=2 / 3$ and (a) $\mu=1.86$, (b) $\mu=2.2$, (c) $\mu=2.5$. Courtesy M. Higuera and J. Porter.

distinct, in contrast to the situation for $\Lambda>0$.

It turns out that the interesting periodic and chaotic behavior which one finds for values of $A$ such as those used in Figs. 4.3b-f is associated with a heteroclinic bifurcation inwolving both $O$ and $U$. The bifurcation sets for this global connection, $U \rightarrow O \rightarrow U$, are shown in Fig. 4.5. In this figure there are three curves of heteroclinic bifurcations which emerge

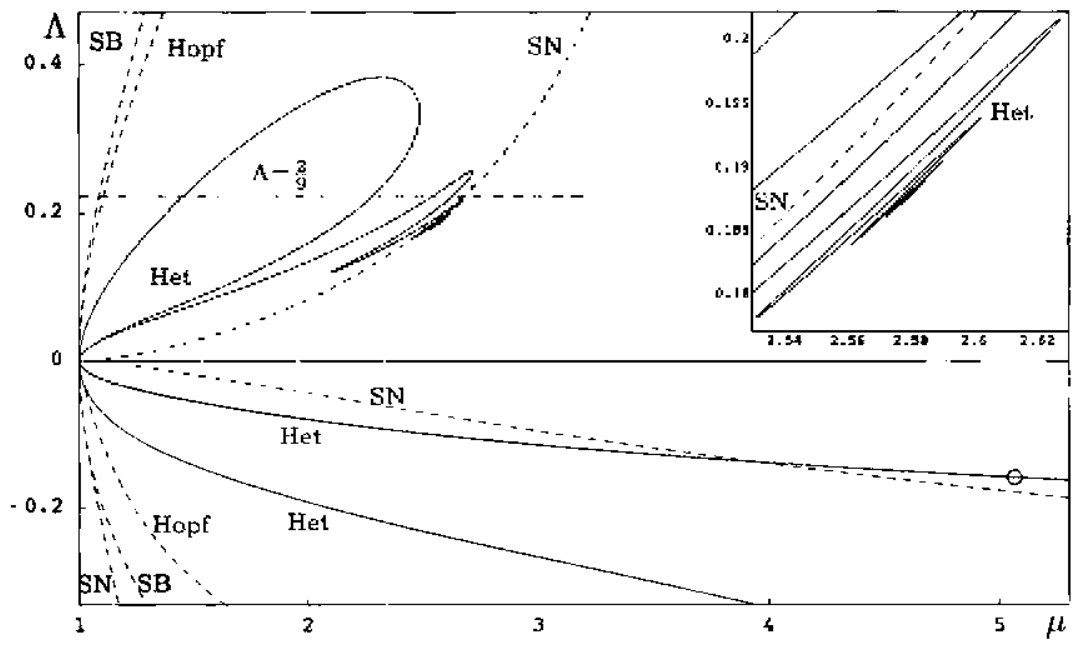

FIGURE 4.5. Heteroclinic (Het) bifurcation sets (solid lines) representing the cycle $U \rightarrow O \rightarrow U$. The inset shows an erlargement of one of these curves near its termination in the codimension-two heteroclinic cycle $U \rightarrow N U \rightarrow O \rightarrow U$. Note that the cut $\Lambda=2 / 9$ passes through four teeteroclinic bifurcations. Courtesy $\mathrm{M}$. Higuera and $J$. Porter.

from $(\mu . \Lambda)=(1,0)$ into the region $\Lambda>0$ and three that emerge into 
the region $\Lambda<0$. For $A>0$ two of these connect up smoothly forming a loop while the third osciliates back and forth an infinite number of times before terminating in a codimension-two heteroclinic bifurcation point at $(\mu, \Lambda) \simeq(2.5803,0.1877)$. The hetcroclinic cycle at this point involves all three types of fixed poirts: $O, U$, and the $N U$ state between the two saddle-node bifurcations on the $N U$ brancl. For $\Lambda<0$ the three curves of heteroclinic bifurcations remain separate (the upper two are almost indistinguishable on the scale of the figure). Two of them continne out to large values of $\mu$ (they have been followed to $\mu>50$ ) while the third wiggles back and forth before terminating in another codimension-two heteroclinic cycle involving $O, U$, and $N U$. This point, $(\mu, 1) \simeq(5.065,-0.159)$, is marked in Fig. 4.5 by a small circle: the wiggles are not visible on this scale. This point differs from the previous codimension-two point for $\Lambda>0$ in a fundamental way because it involves the small amplitude $N U$ state (after the first Hopf bifurcation) whose stable and unstable marifolds are each twodimensional. Thus the codimension-two heteroclinic cycle for $\Lambda>0$ involves three points with one-dimensional unstable manifolds; the connection $O \rightarrow$ $U$ is structurally stable (due to the invariance of the uniform plane) while the connections $U \rightarrow N U$ and $N U \rightarrow O$ are each of codimension-one. For $\Lambda<0$ the connections $O \rightarrow U$ and $N U \rightarrow O$ : are both structurally stable but the third, $U \rightarrow N U$, is itself of codimension two.

Fig. 4.5 also shows the cut $\Lambda=2 / 9$. This cut corresponds to the bifurcation diagram of Fig. 4.3 e and crosses the heteroclinic bifurcation set four times. We use this $A$ walue to investigate further the dynamics associated with this bifurcation. Along this path che first Hopf bifurcation (at. $\mu \simeq 1.106$ ) occurs almost immediately after the birth of the $N U$ branch (see Fig. 4.5). Between this IFopf bifurcation and the leftnost saddle-node bifurcation on the $N U$ branch at $\mu \simeq 2.674$ there are no stable fixed points; in this region one can eisijy find chaotic att.ractors, steh as those shown in Fig. 4.6, as well as a varsety of interesting poriodic solutions (sce Fig. 4.7).

(a)

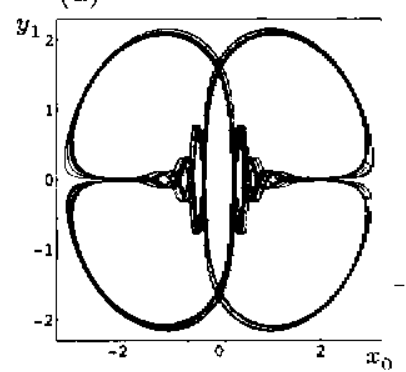

(b)

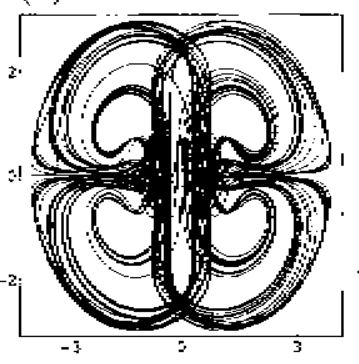

(c)

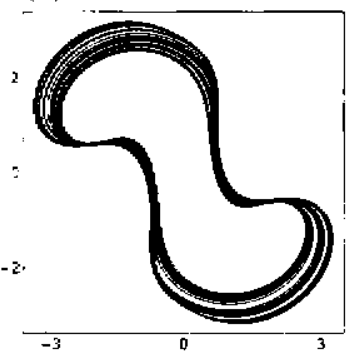

FIgURE 4.6. Chaotic atiractors for $d=0, \alpha=0.1, A=2 / 9$ and (a) $\mu=1.51$, (b) $\mu=2.0$, (c) $\mu=2.54$. Courtesy M. Higuera and J. Porter. 
(a)

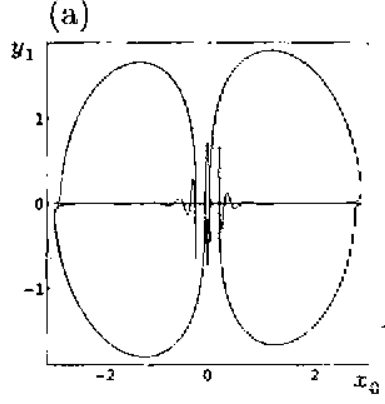

(b)

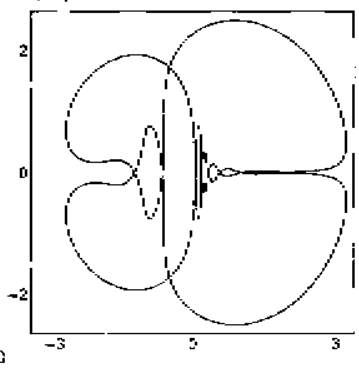

(c)

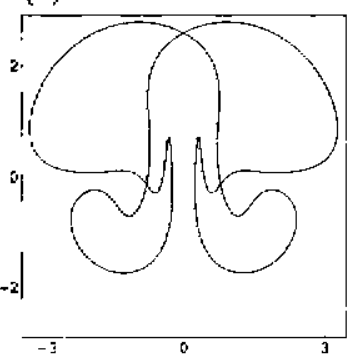

FigurE 4.7. $\mathbb{Z}_{2}$-symmetric periodic attractors for $d=0, \alpha=0.1, \Lambda=2 / 9$ and (a) $\mu=1.41, R_{0} R_{1}$-symmetry; (b) $\mu=1.64 ; R_{1}$-symmetry; (c) $\mu=1.875$, $R_{0}$-symmetry. Courtesy M. Higuera and J. Porter.

Notice that the periodic orbits in Fig. 4.7 have $\mathbb{Z}_{2}$ symmetry, i.e., they are invariant under one of the reflections: $R_{0}, R_{1}, R_{0} R_{1}$. Although thesc particular periodic orbits are somewhat exotic in the sense that they do not belong to one of the basic families of periodic solutions analyzed below but resemble something like the "multi-pulse" orbits identified in perturbations of the Hamiltonian problem) there are also sequences of simpler periodic orbits which come close to both $O$ and $U$. These orbits, characterized by their symmetry (or lack thereof) and by the number of oscillations they experience near $O$, are related in a findariental way to the heteroclinic connection $U \rightarrow O \rightarrow U$. A bifureation diagram obhained by following many of these solutions mumericaliy is tisplayed in Figs. 1.8 , along with four representative orbits. I'his figure shows the period (half-perioc for symmetric orbits) as a function of $\mu$.

I'wo of the tranches shown (the ones with lowest proiod) close on thenselves to form isolas but most. of the solutions terminate in homoclinic: $\left(U_{\perp} \rightarrow U_{\perp}\right)$ gluing bifurrations or hetcroclinic $\left(U_{ \pm} \quad \cdot U_{\mathrm{T}}\right)$ symmetryswitching bifurcations. This is evident from the dramatic increase in period which occurs as the periodic orbits approach the fixed points. In the gluing bifurcations two asymmetric periodic orbits come together (using $U_{+}$ or $U_{-}$) to create a single $R_{1}$-symmetric periodic orbit. In the symmetryswitching bifurcations two $R_{0}$-symmetric periodic orbits transform (using both $U_{+}$and $U_{-}$) into two $R_{0} R_{1}$-symmetric periodic orbits. In this second case the symmetry neither increases nor decreases but switches from one $\mathbb{Z}_{2}$ symmetry to another.

Under appropriate conditions each of these processes is associated, as in the usua! Shil'nikov scenario (Glendinning and Sparrow [1984]; Wiggins [1988]), with cascades of saddle-node and either period-doubling or symmetry-breaking bifurcations; the $\mathbb{Z}_{2}$-5ymmetric orbits must undergo symmetry-breaking prior to any period-doubling bifurcations since such orbits do not (generically) have negatiwe Floquet multipliers (Swift and 
(1)

(2)

(3)

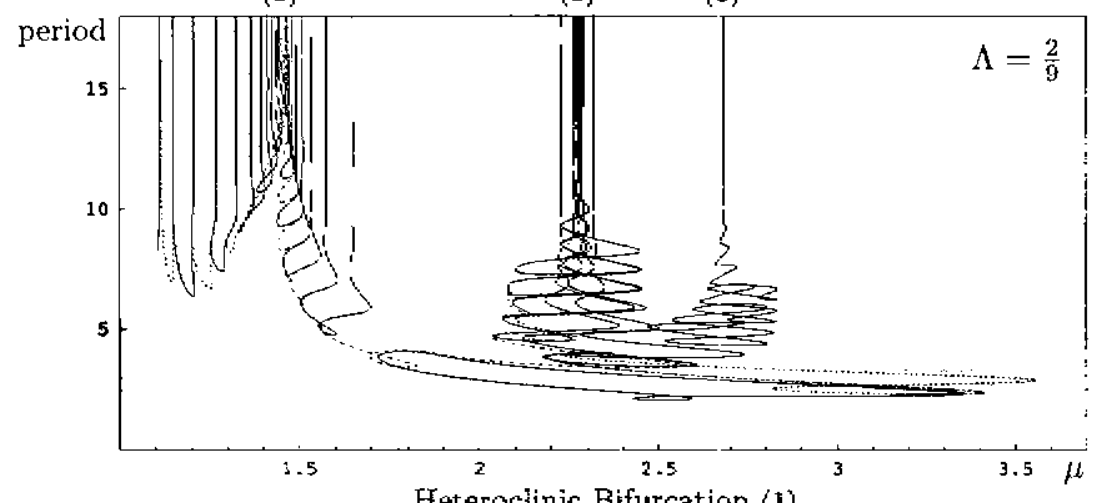

Feteroclinic Bifurcation (1)
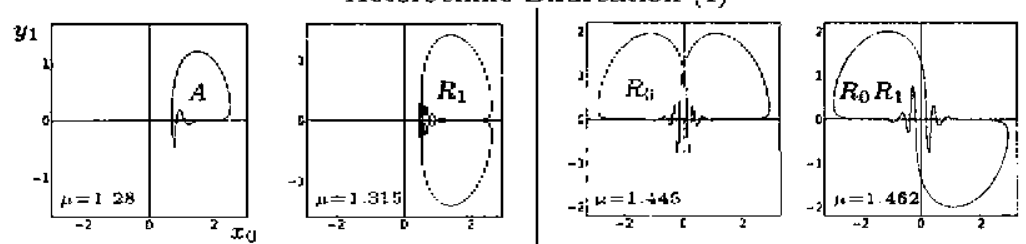

Gluing

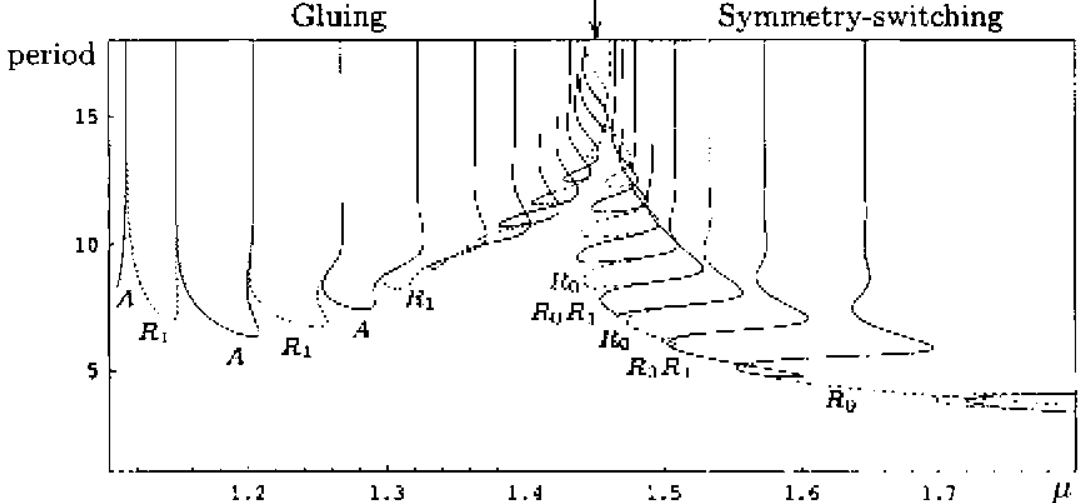

Figure 4.8. Cascardes of gluing $\left(A+A \leftrightarrow R_{1}\right)$ and symmetry-switching $\left(R_{0}+R_{0} \leftrightarrow R_{0} R_{1}+R_{0} R_{1}\right)$ bifurcations for $d=0, \alpha=0.1$ and $\Lambda=2 / 9$. These accumulate from opposite sides on the principal heteroclinic bifurcations, the first two of which, labeled \{1\} and $\{2\}$ : are shown (upper panel). At point (3) there is a homoclinic connection to NU. The lower panel shows an enlargement of the region near point (1). The diagrams show the period (half-period) of asymmetric (symmetric) periodic orbits az a function of $\mu$. Courtesy M. Higuera and J. Porter.

Wiesenfeld [1984]). Note also that the way the two branches (c.g., an asymmetric and an $R_{1}$-symmetric branch) merge with increasing period 
differs from that of the corresponding Shi]'nikov problem in three dimensions with symmetry (Glendinning [1984]). This is because the reflection symmetry in the latter case must be a complete inversion (Tresser [1984]; Wiggins [1988]), while in our case the relevant symmetry $R_{1}$ is not (sec Eq. (4.10)); in particular $R_{1}$ does not act on the swirling part of the flow near $U_{ \pm}$in the plane $c_{1}=0$. In our case the two types of branches oscillate "in phase" around the homoclinic or heteroclinic points as their period increases (cf. Fig. 4.8), while they oscillate "out of plase" in the threedimensional case with inversion symmetry. These differences between the standard situation and ours are a direct consequence of the fact that our two-mode truncation is four-dimensional, allowing new types of connection that are not possible in three dimensions. Notc that in Fig. 4.8 we have only investigated the first two of the main heteroclinic bifurcations (recall that there are four sucl bifurcations when $A=2 / 9$ ) and that there are many periodic solutions (e.g., those of Fig. 4.7) which hawe not been shown; these may form isolas or terminate at other, subsidiary, connections. In short, the full situation is extremely complex.

\subsection{Comparison with the PDE}

Since it is the dynamics of the PDE (4.2) (4.3) that are of ultimate interest, one would like to understand how faithfully their behavior is represented by a truncated set of ordinary differential equations (ODEs). While there is no a priori reason to assume than a finite number of modes can accurately capture the effect of the nonlinear tersms: it tiurrs out that in many problems they ato (Knobloch. Proctor, atel thejss [1993]; Doelman [1991]; Rucklidge and Mathews [1996]). Higuera: Forter, and Knobloch [2002] find numerically that these equations frequently have reflection-symmetric attractors (in $x$ ) antel that these are well described by the restriction to the cosine subspace. In addition, the numerical simulations indicatas that the influnce of the higher modes is often negligible, purlicularly for periodic orbits and chaotic attractors which are approximately heteroclinic. Fig. 4.9 shows that the heteroclinic behavior found within the two-mode model (4.8)-(4.9) also occurs in the full PDE.

To examine the influence of higher modes $(n>1)$ on the dynamics we have computed $\left|c_{0}\right|,\left|c_{1}\right|$, and $\sum_{n=2}^{N} \mid c_{n}$ as functions of time, after first allowing transients to die away. The solutions in Fig. 4.10 represent typical chaotic attractors that can be found for $A=2 / 9$ and $1.5 \lesssim \mu \lesssim 2.8$, together with the time series representing their harmonic content. Notice that in all cases the amplitude of the higher modes (bold curves in the righthand set of panels) remains small, indicating that these modes do not play a significant role in the dynamies.

While such a low-dimensional description is not unexpected for small amplitudes (i.e., near onset at $\mu=1$ ) Eqs. (4.2)-(4.3) continuc to be described by the two mode truncation even relatively far from the primary bifurcation. Notice that, e.g., for $A=2 / 9$ and $\mu \gtrsim 1.875$ the uniform states are 

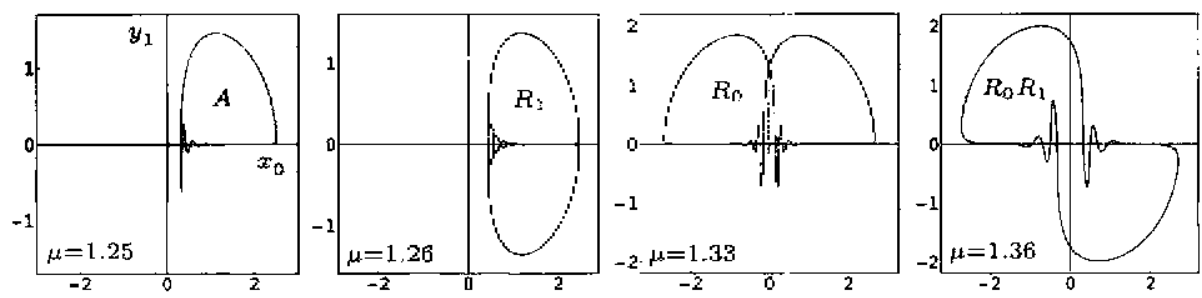

FIgure 4.9. Stable periodic orbits of the PDE (4.2)-(4.3) with different symmetries for $\Lambda=2 / 9$. Gluing and symmetry-switchirg bifurcations, as in the ODEs, appear to be present. Courtesy h. Higuera ard I. Porter.

unstable to at least two nomuniform modes and one might therefore suppose that a two-mode truncation will be of dubious validity. However, we often find that the system (4.8)-(4.9) continues to apply (see Figs. 4.10b,c). This increased range of validity is likely due to the prominence of the heteroclinic bifurcation since for orbits which are approxinately heteroclinic the potentially complicated dynamics of the full PDE are controlled mainly by symmetries and by the local properties of the fixed points $O$ and $U$ where most time is spent; recall that $O$ and $[\dot{y}$ are the same in both the PDE (4.2)-(4.3) and the ODE model (4.8)-(4.9). Also important is the fact that die to the spatial avoraging of the forcing term in Eq. (4.2) the origin is always stable with respect to nonuniform modes. The higher modes are thus quickly damped ujucter the attractirg infuence of the trivial state. We conclude that the evjonte dow-limensional behavior of the PDF (4.2). (4.3) is related to the presence of the beterolinic bifurcation involving the origin and its associatend cabcades. Wheriever one is relatively close to these bifurcations in parameter space (sec Fig- 4.5 \} the dynamics will typically be dominated by the maxy periodic and chutic attractors associated with then. For parameter values outside of this rogime (c.g, $\mu \geq 3$ when $\Lambda=2 / 9$ ) the dynamics are no longer heteroclinic and hence are more likely to involve other modes.

When $\Lambda=2 / 3$, the value used in Hartel, Knobloch, and Vega [2000] for Fig. 4.1, the heteroclinic bifurcation does not actually occur (see Fig. 4.5), but the dynamics may nonctheless be dominated by the various periodic orbits and related chaotic attractors which exist in nearby regions of parameter space; ghing bifurcations still occur even though the full cascade does not. Fig. 4.11 shows several chaotic attractors for $A=2 / 3$ demonstrating that the dynamics are again dominated by the first two modes. As for $\Lambda=2 / 9$, this low-dimensional beharior does not hold for all values of $\mu$ and the two-mode ODE model eventually fails. But in contrast to the case $\Lambda=2 / 9$, when $\Lambda=2 / 3$ this failure can arise for two reasons. The first failure of Eqs. (4.8)-(4.9) is due to a. $\mathcal{R}$ symmetry-breaking bifurcation, which occurs at $\mu \sim 3.4$. In this case it is not the two-mode nature of the model 
(a)
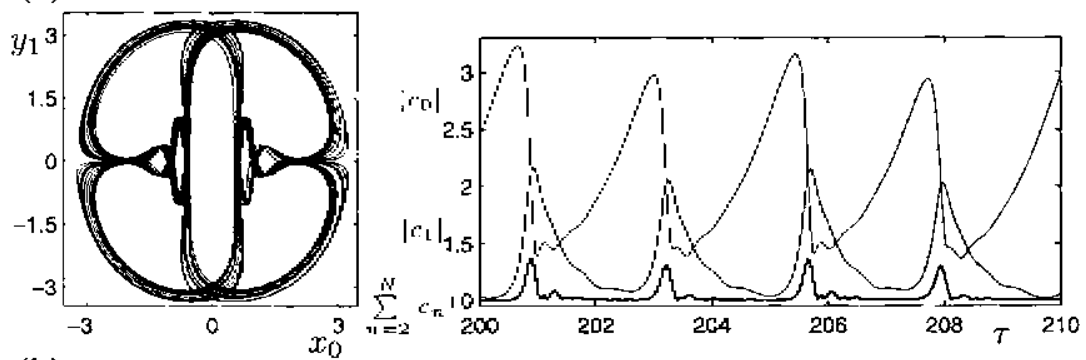

(b)
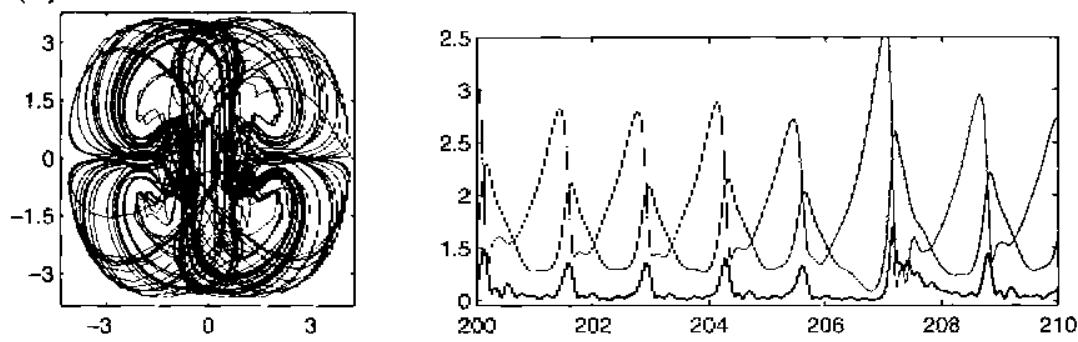

(c)
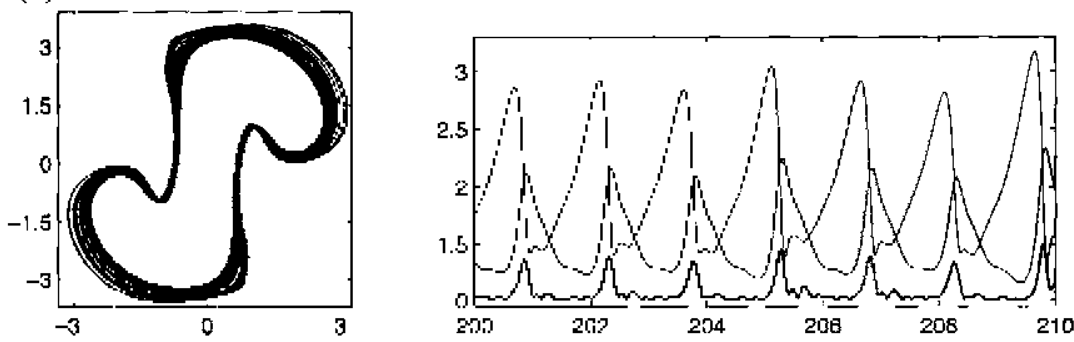

Figure 4.10. Relative importance of the Fourier componente for $A=2 / 9$ : (a) chaotic attractor at $\mu=1.51$, (b) at $\mu=2.0,(\mathrm{c})$ at $\mu=2.8$. The lines $(\longrightarrow)$ correspond to $\left|c_{\mathrm{Q}}\right|$, (Higuera and J. Porter.

that becomes inappropriate (the uniform state does not lose stability to the $n=2$ mode until $\mu \simeq 4$. (093) but the restriction to the cosine subspace. Fig. 4.12a shows a solution, which possesses low-dimensional character but is not reflection-symmetric and is therefore not contained within the system (4.8)-(4.9). After a narrow interval $\{3.4 \lesssim \mu \lesssim 3.46)$ the dynamics recover their reflection-symmetric character, and subsequently (see Fig. 4.1) a second window of stable uniform states appears for $3.5 \lesssim \mu \lesssim 4.3$. At $\mu \sim 4.3$ the system becomes abruptly chzotic. with many modes partaking in the dynamics. This situation, however, does not persist uniformly as $\mu$ increases further. For example, at $\mu=4.65$ the trajectories spend a long 
(a)
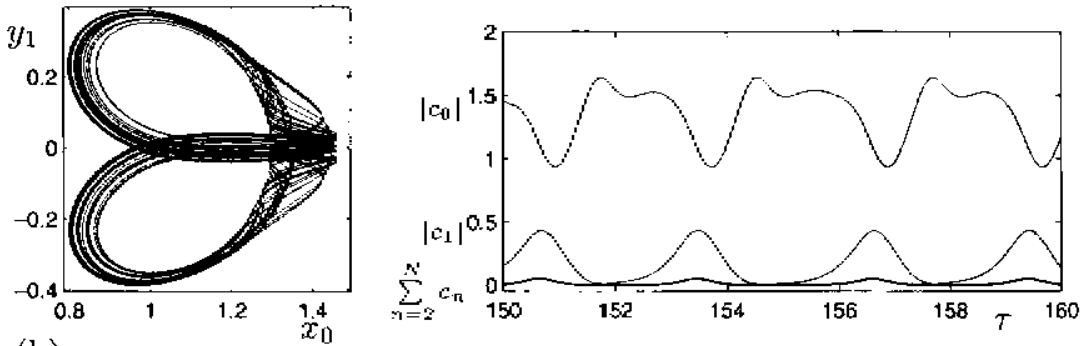

(b)
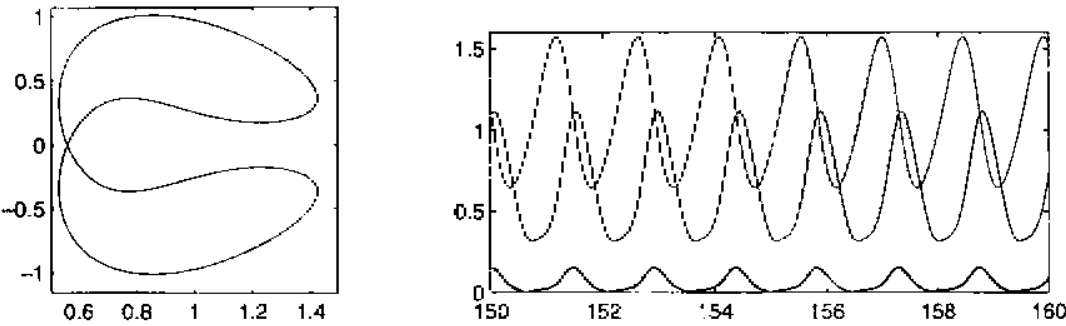

(c)
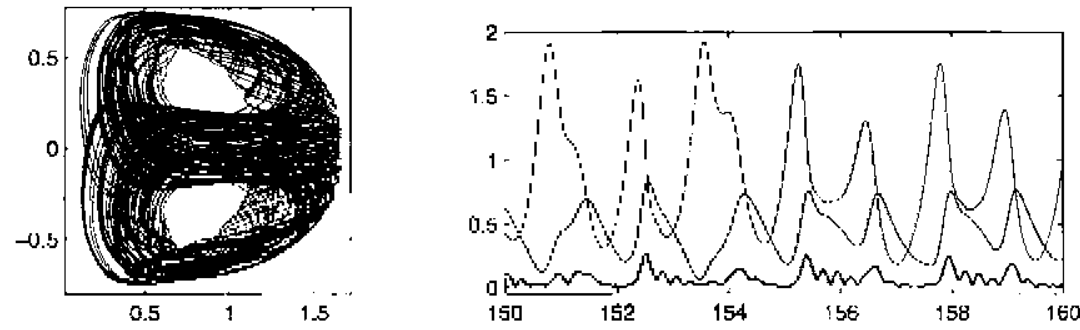

Figdre 4.11. Relative importance of the different fouries compoments when $\Lambda=2 / 3$ for chaotic attractors at: (a) $\mu=1.85$, (b) $\mu=1.925$, and (c) $\mu=3.2$. The lines (Courtesy M. Higlera and J. Porter.

time near the invariant even subspace ( $c_{n}=0$ if $n$ is odd), occasionally coming under the influence of unstable periodic orbits in this subspace and being briefly ejected from the even subspace (see Fig. 4.13). These excursions are associated with episodic phase drift of the solution (type I drift in the terminology of Martel, Knobloch, and Yega [2000]). This interesting behavior is reminiscent of the so-called blowout bifurcation (see, e.g., Ashwin, Buescu, and Stewart [1996]). In the present case the attractor is completely contained in the even subspace (with dynamies dominated by the first two even modes, $n=0,2$ j over a moderately large interval, $5.0 \lesssim \mu \lesssim 6.5$, but loses stability, apparently in the above manner, as $\mu$ decreases below $\mu \simeq 5.0$. We remark that blowout bifurcations provide a 
(a)

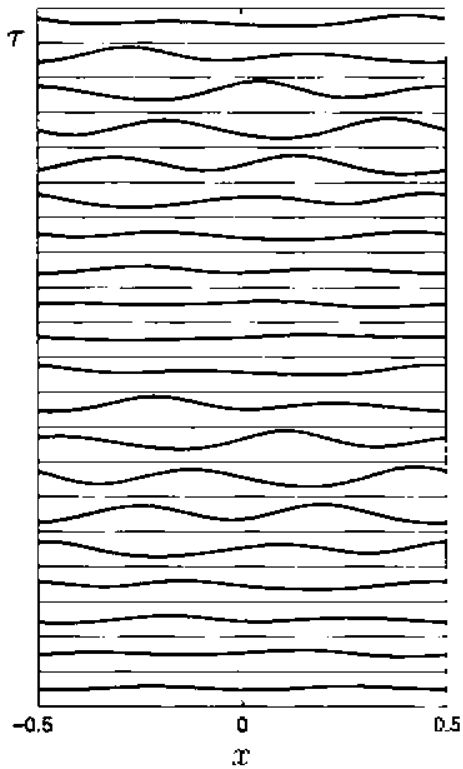

(b)

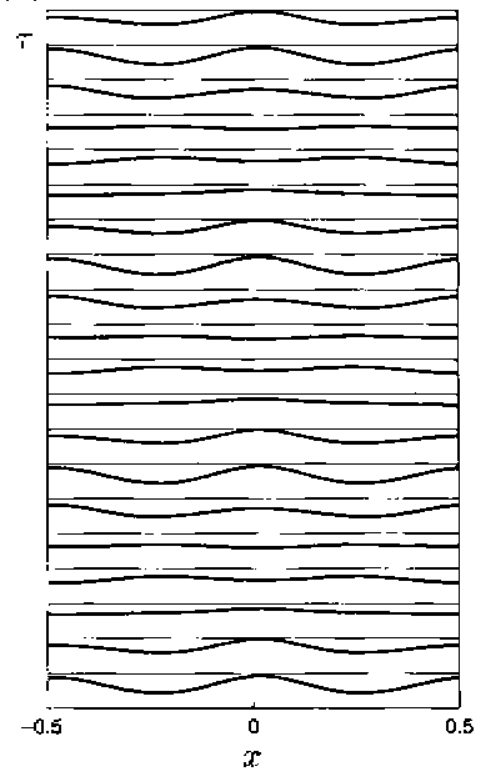

FIGURE 4.12. Space-time diagrams corresponding to (a) a quasiperiodic attractor without reflection symmetry. $A=2 / 3$ and $\mu=3.4$; and (b) a periodic attractor with reflection symmetry, $h=2 / 9$ and $\mu=3.46$. Courtesy $M$. Higuera and $J$. Porter.

general mechanism by whikh at tractors in invariant subspaces lose stability with respect to perturbations out of the subspace.

\section{Concluding Remarks}

In this paper we have summarized the results of a systematic derivation of the amplitude equations describing the evolution of slowly varying wavetrains on the surface of a nearly inviscid liquid excited by small amplitude vertical vibration of its container. Because of the presence of oscillatory viscous boundary layers along the rigid boundaries and the free surface viscous mean flows are driven in the largely inwiscid interior of the fluid. These augment any inviscid mean flows that may be present and the two together interact with the parametrically excited waves producing them. This nontrivial interaction between the mean flows and the waves is a consequence of the presence of the hydrodynamic modes which decay, for $C_{g} \ll 1$, more slowly than gravity-capillary waves, and hence are easily excited by the oscillations. The resulting equations, albeit still complex, provide a significant simplification of the original problem in that the boundary conditions are now applied at the undeformed surface, and the fast oscillation frequency 


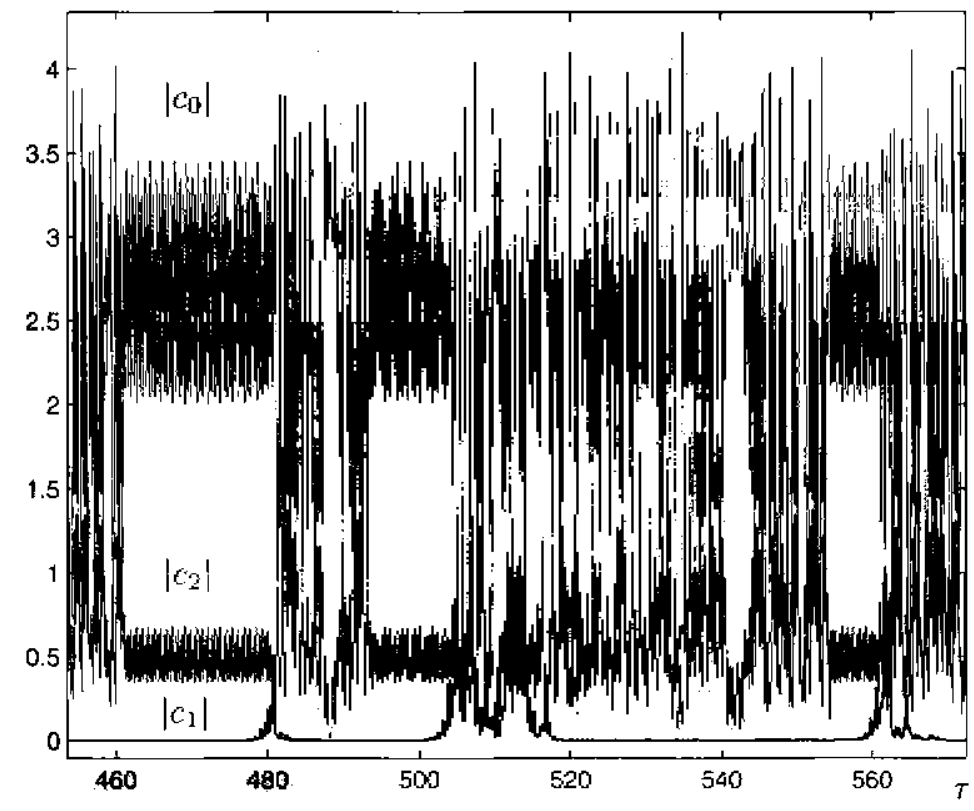

Figure 4.13. Norm of the first three modes versus $\tau$ for $A=2 / 3$ and $\mu=4.65$. The thin, medium, and thick lines denote $\left|c_{0}\right|,\left|c_{2}\right|$, and $\left|c_{1}\right|$, respectively. Note the episodic excitation of the mode $c_{2}$. Courtesy M. Higuera and J. Porter.

associated with the vibration of the container lass been elininated. As part of the analysis explinit expressions for all the coefficients are obtained, as are explicit conditions for the validity of the resulting equations (Vega, Knobloch, and Martel i2001 $^{\circ}$ ). As such the resulting equations represent a novel system for the study of pattern formation and subsequent instabilities of the resulting patterns wia the excilation of medn flows.

In certain specific cases these cquations can be simplifed further. We discussed one such case, in which the mean flow decouples from the amplitude equations for the left- and right-traveling waves. The remaining cquations are still not trivial, in that they are nonlocal and include both dispersion and damping, although no wavenumber-dependent dissipation. Equations of this type were studied by Martel: Knobloch, and Vega [2000] and provide perhaps the simplest description of the Faraday system in an extended domain under precisely stated conditions. It is important to emphasize that this description differs from those obtained by ad hoc procedures. In particular, the usual approach of formulating the problem as an inviscid one at leading order, and adding some damping after the fact to mimic the role of viscosity fails on two levels: it onits the basic mechanisms that drive the (viscous) mean flow (Schlichting [1932]), and it omits the back-reaction of this flow on the waves that are responsible for it. Even the simplest 
description of the Faraday system that results includes nonlocal terms in the amplitude equations whose origin can be traced to the fact that amplitude inhomogeities are advected at the group velocity on a timescale that is much faster than the timescale on which the waves equilibrate. An additional nonlocal contribution arises from the requirement that mass be conserved (Pierce and Knobloch [1994']. Since the Reynolds number of the associated flow can be (jndeed must be) substantial the equations for this flow must in general be solved numerically as already done in other circumstances (Nicolás, Rivas, and Vega. [1997, 1998]).

A careful examination of the analysis that led us to equations (2.11)(2.23) shows that these in fact apply under the conditions

$$
k\left(\left|\psi_{x}\right|+\left|\xi_{y}\right|\right) \ll \omega_{1}, \quad|f|+\left|f_{x}\right| \ll 1, \quad L^{-1} \ll k,
$$

or equivalently,

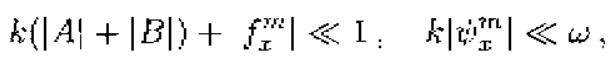

and the condition

$$
L \ll v_{g} l(\delta+d+. \alpha s \mu) .
$$

Here $v_{g}$ is the (nondimensionai) group welocity of the surface waves, defined in (2.14), $\alpha_{5}$ is given in (2.16) and we assumed that the smallest spatial scale is $k^{-1}$. The condition (5.1) can be stated succinctly as requiring that the nonlinearity be weak and the aspect ratio of the system be large compared to the nondinensional wavelength of the surface waves; the condition (5.3) requires that the terms acomutirg for inertin ared propkgation at the group velocity in the anplitude equations (2.I1) (2.12) be nuch larger than the remaining terms. In addition, the matirements

$$
(1-S) k^{2}+S h^{1} \geqslant C_{y}^{2}, \quad h^{\frac{3}{2}}\left(1-S+S k^{2}\right)^{-\frac{1}{2}} \& C_{g}^{x-1},
$$

or equivalently,

$$
C_{g} \ll w, \quad C_{g}^{\frac{1}{2}} \omega^{\frac{3}{2}} \ll 1-S+\left(S \omega / C_{g}\right)
$$

are imposed implicitly both on the carrier wavenumber $k$ as well as on all wavenumbers associated with the (viscous) mean flow. These conditions guarantee that the thickness of the associated boundary layers will be small compared to the depth (if $k \ll 1$ ) or compared to the wavelength (if $k \gg 1$ ), see Fig. 2.2. Since the lowest wavenumber of the mean flow is $k=2 \pi / L$ the condition (5.4) implies, in particular, tiat

$$
(1-S) L^{-2}+(2 \pi)^{2} S L^{-4} \gg C_{3}^{2}
$$

Several additional assumptions appear in the course of the analysis (Vega, Knobloch, and Martel [2001]). 
It is evident that strictly inviscid treatments of the problem and the powerful techniques that are arailable for such treatments miss qualitatively important properties of vibrating systems. Similar issues arise in the theory of vibrating liquid bridges (Nijoolás and Vega [1996]) and related systems (Higuera, Nicolas, and Yega [2000) : where mean flows generated in the viscous boundary layers can be used to control the amplitude of any convection that may be present. Whether the approach described here for the Faraday system will yield a quantitatively precise description of existing experiments on the Faraday system with nearly inviscid fluids (Ezerskii, Rabinovich, Reutor, and Starobinets 1986]; Douady, Fauve, and Thual [1989]; Tufillaro, Ramshankar: and Gollub [1989]; Kudrolli and Gollub [1997]) remains to be seen, howewer. Any experiments in a narrow annulus will suffer from effects due to oscillatory boundary layers at the lateral (radial) boundaries which are difficult to minimize. Likewise precise experiments on liquid bridges are difficult under terrestrial conditions, and stability predictions of the type given by Kruse, Mahalov, and Marsden [1999] remain to be confirmed.

The relation between the type of theory described here and earlier work (Kovačič and Wiggins [1992; Haller and Higgins [1993, 1995a,b]) on the origin of complex dynamics in the forced weakly damped nonlinear Schrödinger equation is also of interest. This work focused on the near-Hamiltonian limit and exploited generalizations of the Mil'nikov theory to PDEs to establish the presence of a variety of multipulse orbits homoclinic or heteroclinic to a slow manifold. In contrast. our approach has focused on the dynamics smbstantially faxther from this linit. Althongh much of the dynamical behavior found numcrically in the noulacal parametrically forced damped nonlinear Solhrötinger equation derived here cond be understood in detail using a two-mole model sysicm, the refation of the cascades of gluing and symmetry-switching bifurcations that appear to be responsible for it to the near-IIaniltunian dyndmics andyzed for this class of systems by Kovacic and Wiggins 1092] and Haller and Wiggins [1993, 1995a., $]$ remains to be examined. Indeed, because of the parametric nature of the forcing (and in particular the resulting symmetry $C \rightarrow-C$ ) the behavior found here bears a greater resembiance to that discussed by Rucklidge and Matthews [1996] in their study of the dynamics of the shearing instability in magnetoconvection than to the damped nonlinear Schrödinger equation with direct forcing. Like our system the former has $D_{2}$ symmetry and exhibits global bifurcations involving both the origin (corresponding to the conduction state) and the convective state $\mathrm{SS}$. The latter state is reflectionsymmetric and can undergo a pitchfork bifurcation to a tilted convection state STC. From a symmetry point of view these states play the same role as $O, U$ and $N U$ in our problem. The essentjal difference between our system and that studied by Rucklidge and hattinews lies in the fact that in our case the leading stable eigenvalues of both $O$ and $U^{\prime}$ are complex (the former in the $c_{0}=0$ subspace, and the latter in the $c_{1}=0$ subspace). The dynamical 
behavior that results is new and is discussed in detail in Higuera, Porter, and Knobloch [2002] and Porter [2001]. Truncated Galerkin expansions of the type that led us to this behavior have, of course, also becn used to study the effect of direct forcing on the sine-Gordon equation, a system closely related to ours. Here, too, the study of the finite-dimensional system proved of substantial help in understanding the PDE simulations (Bishop, Forest, McLaughlin, and Overman [1990]; McLaughlin, Overman, Wiggins, and Xiong [1996|). It should therefore not come as a complete surprise that the two-mode model constructed here captures so much of the behavior found numerically in the PDE (4.2)-(4.3) by Hartcl, Knobloch, and Vega [2000].

Acknowledgments: We are wery grateful for long-term assistance from our colleagues M. Higutera, C. Hartel, J. Nicolás and J. Porter with whom the rcsults reported here wcre obtained. This work was supported in part by the National Aeronautics and Space Administration under Grant NAG32152 and by the Spanish Dirección General de Enseñanza Superior under Grant PB97-0556.

\section{References}

Abarbanel, H. D. I., D. D. Holm, J. E. Marsden, and T. S. Ratiu [1986], Nonlinear stability analysis of stratified fluid equilibria, Phis. Trans. Ron. Soc. London $A, 318: 349109$.

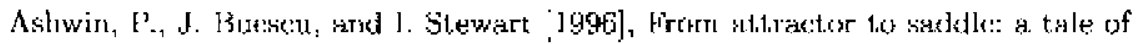
transverse instability, Nonlinearity, 9:703-738.

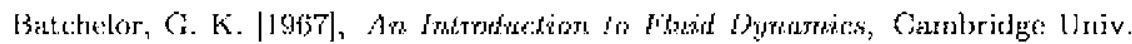
Prestis.

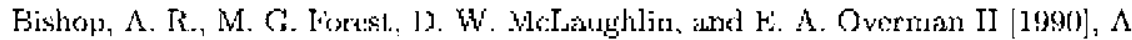

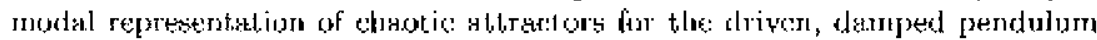
chain, Phys. Lett. A, 144:1j-25.

Chorin, A. and J. E. Marsden [1979], A Mathematical Introduction to Fluid Mechanics Springer-Verlag-

Craik, A. D. D. (1982): The drift velocity of water waves, J. Fluid Mech., 116:187205.

Davey, A., L. M. Hocking, and K. Stewartson [1974, On nonlinear evolution of three-dimensional disturbances in plane Poiseuille flow, J. Fluid Mech,63:529536.

Davey, A. and K. Stewartson 1974, On three-dimensional packets of surface waves, Proc. R. Soc. Londons, Ser. A, 338:101-110.

Doedel, E. J., A. R. Champneys, T. F. Fairgrieve: Y. Kuznetsov, B. Sandstede, and X. J. Wang [1997], ALITO 97: Contination and bifurcation software for ordinary differential equations available via FTP from directory $\mathrm{pub} /$ doedel/auto at ftp.cs.corjcordia.ca). 
Doelman, A. [1991], Finite-dimensional models of the Ginzburg-Landau equation, Nonlinearity, 4:231-250.

Douady, S., S. Fauve, and O. Thual [1989]: Oscillatory phase modulation of parametrically forced surface waves, Europhys. Lett., 10:309 315.

Duan, J., H. V. Ly, and E. S. Tit, [1996]: The effects of nonlocal interactions on the dynamics of the Ginzburg Landau equation, Z. angrw. Math. Phys., $47: 432-455$.

Ermentront, B. [2000]: XPPAUT, Dynamical systems software with continuation and bifurcation capabilities (available via FTP from directory/pub/bardware at ftp.math.pit.edu).

Ezerskii, A. B., M. I. Rabinorich, Y. P. Reutov, and I. M. Starobinets [1986], Spatiotemporal chaos in the parametric excitation of a capillary ripple, Sov. Phys. JETP, 64:1228-1236.

Fanve, S. [1995], Parametric instabilities, In G. Martínez Mekler and T.H. Seligman, editors, Dymarnics of sronlimear and Disonderenl Systems, pp. 67115. World Scientific.

Ghidaglia, J. M. [1988], Finite-dimensional behaviour for weakly damped driven Schrödinger oquations, Anr. Inst. H. Poncaré - Arral. Non-Linéaire, 5:365405.

Glendinning, P. [1984], Bifurcations near homoclinic orbits with symmetry, Phys. Lett. A, 103:163-166.

Glendinning, P. and C. Sparrow' 1984], Local and global behavior near homoclinic orbits, f. Stat. Phys. 35:645-696.

Goubel, O. [1996], Regularity of inlutathe for a vieakly dampod nonlinenr Schrödinger equation, Appl. Arusd, 60-99-119.

IIaller, G. and S. Wiggins [1993]: Orbits homoclisir: to resonances: the Hamiltonian case, 1 thysica $D, 66: 298-346$.

Haller, G. and S. Wiggien |1995aj: h-pulse homodinic ortrits in pexturbations of resonant Hanilionian systense. Amh. Fat. Ateth. Aral., 130:25-101.

Ilaller, G. and S. Wigfims [19y5b], Multi-pulse jumping orbits and homoclinic trees in a modal truncation of the damped-forced nonlinear Schrödinger equation, Physica D, 85:311 347.

Hansen, P. L. and P. Alstrom [1997]. Perturbation theory of parametrically driven capillary waves at low viscosity, J. Fhidd Mech, 351:301-344.

Henderson, D. M. and J. W. Miles [1994. Surface-wave damping in a circular cylinder with a fixed contact line, J. Flusd Hech., 275:285 299.

Higuera, M., J. A. Nicolás, and J. M. Yega [2000], Coupled amplitude-streaming flow equations for the evolution of counter-rotating: nearly-inviscid surface waves in finite axisymmetric geometries, Preprint.

Higuera, M., J. Porter, and E. Knobloch [2002], Heteroclinic dymamics in the nonlocal parametrically driven Schrödinger equation, Physica $D, 162: 155-187$.

Holm, D. D., J. E. Marsden, T. S. Ratiu, and A. Wieinstein [1985], Nonlinear stability of fluid and plasma equilibria. Phys. Rep., 123:1-116. 
Holm, D. D., J. E. Marsden, and T. S. Ratiu [1986], Nonlinear stability of the Kelvin-Stuart cat's eyes flow, in Lects. in Appl. Wfoth., 23:171-186.

Kakutani, T. and K. Matsuucki [1975], Effect of viscosity on long gravity waves, J. Phys. Soc. Japan, 39:237-246.

Knobloch, E. and R. Pierce [1998?. On mean flows associated with travelling water waves, Fluid Dyn. Res., 22:61- $\mathrm{i} 1$.

Knobloch, E., M. R. F. Proctor, and N. O. Wieiss [1993], Finite-dimensional description of doubly diffusive convection. in Turbulence in Flud Flows: $A$ Dy. namical Systems Approach, G.R. Sel], C. Foias, and R. Temam (eds), SpringerVerlag, Now York, IAA Volumes in Mathematies and its Applications 55, pp. $59-72$.

Knobloch, E. and N. O. Weiss [1981], Bjfurcations in a model of double-diffusive convection, Phys. Lett. A, $8 \overline{3}: 127-130$.

Kovačič, G. and S. Wiggitus 1992], Orbits bomoclinic to resonanes, with an application to chaos in a model of the forced and damped sine-Gordon equation, Physica $D, 57: 185 \cdot 225$.

Kruse, K.-P., A. Mahalov, and J. F. Marsden '1999], On the Hamiltonian structure and three-dinensional instabilities of rotating liquid bridges. Fluid Dyn. Res., 24:37-59.

Kudrolli, A. and J. P. Gollub [1997, Patterns and spatio-temporal chaos in parametrically forced surface waves: A systematic survey at large aspect ratio, Physica $D, 97: 133-154$.

Leibovich, S. [1983]. On wave-current interaction throries of Langmuir circulations Ann. Rev. Fluid Mech, 15:391-427.

Lewis, D., J, E. Marsden, R. Murstgontt:ry: aud T. S. R.atiul [1986], The Hamilto

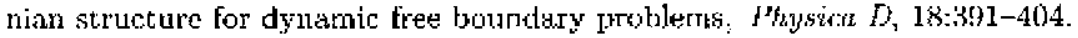

Longuet-Higgins, M. S. 'I953, Mist trilrerrert in waler waves, Phil. Trans. $R$. Soc. London, Ser, $A, 245: 535581$.

Marsden, J. F. and P. J. Murrisurs $|1984|$, Noncanonical Hamitonian field theory

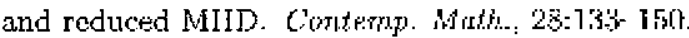

Martel, C. arm $F$. Knoljuch [l997: Damping of nearly inviscid water waves, Phys. Rev. E, 56:5544-5048.

Martel, C., E. Knobloch, aud J. M. Yega [2000], Dynamics of counterpropagating waves in parametrically forced systems. Pliysica $D, 137: 94-123$.

McLaughlin, D. W., E. A. Overnar: II. S. wiggins, and C. Xiong [1996], Honoclinic orbits in a four-dimensional model of a perturbed NLS equation: A geometric singular perturbation study, in Dynamics Reported, vol. 5, SpringerVerlag, New York; p. 190.

Miles, J. W. [1993], On Faraday waves, J. Fluid Mech. 248:671-683.

Miles, J. and D. Henderson [1990], Parametrically forced surface waves, Ann. Rev. Fluid Mech., 22:14\$-165.

Nicolás, J. A., D. Rivas, and J. Mi. Yega !1997], The interaction of thermocapillary convection and low-frequency vibration in nearty-inviscid liquid bridges, $Z$. Angew. Math. Phys., 48:389-423. 
Nicolás, J. A., D. Rivas, and J. M. Yega 1998], On the steady streanning flow due to high frequency vibration in nearly-inwiscid liquid bridges, J. Fluid Mech., $354: 147 \cdot 174$.

Nicolás, J. A. and J. M. Vega [1996], Wheakly nonlinear oscillations of axisymmetric liquid bridges, J. Fluid Wech. 328:95 100.

Oliver, M. and E. Titi [1998. Analyticity of the attractor and the number of determining modes for a weakly damped driven noulinear Schrödinger cquation, Indiana Univ. Math. J., 47:49-73.

Phillips, O. M. [1977]: The Dymamics of the Upper Ocean, Cambridge Univ. Press.

Pierce, R. D. and E. Knobloch [1994]: On the modulational stability of trave]ing and standing water waves, Phys. Fluids, 6:1177-1190.

Porter, J. B. [2001], Global bifurcations with symmetry. Ph.D. Thesis, University of California at Berkeley-

Riecke, H., J. D. Crawford, and E. Knoblock [1988], Time-modulated oscillatory convection, Phys. Rev. Lett., 61:1942-1945.

Rucklidge, A. M. and P. C. Matthews [1996.: Analysis of the shearing instability in nonlinear convection and magretoronvection. Nonlinearity, 9:311-351.

Schlichting, H. [1932], Berechnung ebener periodischer Grenzschichtströmungen, Phys. Z., 33:327-335.

Swift, J. W. and K. Wiesenfeld 1984], Suppression of period doubling in symmetric systems, Phys. Rev. Leti., 52:705 708.

Tresser, C. [1984], About some theorems by L.P. Shilnikov, Ann. Inst. Henri Poincaré - Phys. Theorique, 40:440-461.

Tufillaro, N. B., R. Ramshankar, and J. P. Gollub [1989], Order-disorder transition in capillary ripples: Phys. Fiec. Lett. 602:422-425.

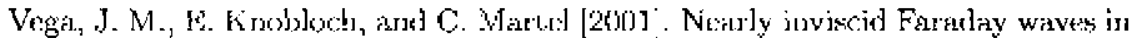

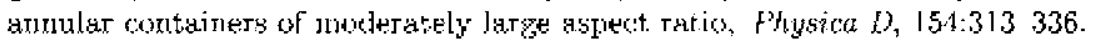

Wang, $\mathrm{X}$. [194i], An energy equation for the weakly damped driven nomlincar Schrödinger equations and jos application to their attractors, Physica $D$, 88: $167 \quad 175$.

Wiggins, S. [1988], Global Bifturcations and Chaos: Analytical Methods, SpringerVerlag, New York. 